\title{
OPTIMAL CONTROL FOR STOCHASTIC VOLTERRA EQUATIONS WITH COMPLETELY MONOTONE KERNELS*
}

\author{
S. BONACCORSI ${ }^{\dagger}$, F. CONFORTOLA ${ }^{\ddagger}$, AND E. MASTROGIACOMO $\ddagger$
}

\begin{abstract}
In this paper, we study a class of optimal control problems for stochastic Volterra equations in infinite dimensions. We are concerned with a class of stochastic Volterra integrodifferential problem with completely monotone kernels, where we assume that the noise enters the system when we introduce a control. We provide a semigroup setting for the problem, by the state space setting; the applications to optimal control provide other interesting results and require a precise description of the properties of the generated semigroup. In our stochastic optimal control problems, the drift term of the equation has a linear growth in the control variable, the cost functional has a quadratic growth, and the control process belongs to the class of square integrable, adapted processes with no bound assumed on it. Our main results are the existence for the optimal feedback control, the identification of the optimal cost with the value $Y_{0}$ of the maximal solution $(Y, Z)$ of the backward stochastic differential equation, the existence of a weak solution to the so-called closed loop equation and, finally, the construction of an optimal feedback in terms of the process $Z$.
\end{abstract}

Key words. stochastic Volterra equations, optimal control problems, quadratic cost

AMS subject classifications. 45D05, 93E20, 60H30

DOI. $10.1137 / 100782875$

1. Introduction. The purpose of this paper is to present some new results of stochastic optimal control problems arising in the context of integro-differential dynamical systems. In recent years, many investigations have been carried out concerning HJB equations in connection with optimal control of nonlinear infinite dimensional stochastic systems; to the best of our knowledge, this paper is the first that attempts to use this approach to control a system whose dynamic is perturbed by the memory term.

In this section we briefly outline the main steps of our construction; this is meant as a guideline for the reader, who will find in later sections all the details.

Throughout the paper we assume that $H, \Xi$, and $U$ are real separable Hilbert spaces. In this paper we model a realistic situation where the control itself is perturbed by a noise. We are thus concerned with the following class of controlled stochastic integral Volterra equation on $H$ :

$$
\begin{aligned}
\frac{d}{d t} \int_{-\infty}^{t} a(t-s) u(s) \mathrm{d} s & =A u(t)+g(t, u(t))[r(t, u(t), \gamma(t))+\dot{W}(t)], \\
u(t) & =u_{0}(t), \quad t \leq 0,
\end{aligned}
$$

where $\gamma$ is the control process, which is assumed to take values in $\Xi$, and $W(t)$ is a cylindrical Wiener process taking values in $U$.

* Received by the editors January 19, 2010; accepted for publication (in revised form) December 15, 2011; published electronically March 22, 2012.

http://www.siam.org/journals/sicon/50-2/78287.html

†Università di Trento, Dipartimento di Matematica, Via Sommarive 14, 38050 Povo (Trento), Italia (stefano.bonaccorsi@unitn.it).

${ }_{\ddagger}^{\ddagger}$ Politecnico di Milano, Dipartimento di Matematica F. Brioschi, Piazza Leonardo da Vinci 32, 20133 Milano, Italia (elisa.mastrogiacomo@polimi.it, fulvia.confortola@polimi.it).

748 
The control problem consists of minimizing a cost functional of the form

$$
\mathbb{J}\left(u_{0}, \gamma\right)=\mathbb{E} \int_{0}^{T} l(t, u(t), \gamma(t)) \mathrm{d} t+\mathbb{E}[\phi(u(T))] .
$$

In order to handle the control problem, we first restate (1.1) in an evolution setting by the state space setting first introduced in $[28,14]$ and recently revised, for the stochastic case, in $[24,4,5]$. Thus we obtain a stochastic evolution equation in a (different) Hilbert space $X$ :

$$
\begin{aligned}
\mathrm{d} v(t) & =[B v(t)+(I-B) P F(t, v(t), \gamma(t))] \mathrm{d} t,+(I-B) P G(t, v(t)) \mathrm{d} W_{t}, \\
v(0) & =v_{0},
\end{aligned}
$$

where the coefficients $F$ and $G$ involve the maps $g$ and $r$ of the Volterra equation.

Such an approach, called state space setting, has the following interpretation. On a different Hilbert space $X$, the internal state of the system at time $t$ is recorded into a random variable $v(t)$, which contains all the information about the solution up to time $t$. The state space $X$ is quite large and does not have a direct interpretation in terms of the original space $H . B: D(B) \subset X \rightarrow X$ is the operator which governs the evolution from past to future. It is proved that $B$ generates an analytic, strongly continuous semigroup on $X$. We again appeal to interpolation theory in order to define the spaces $X_{\eta}=(X, D(B))_{\eta, 2}$. The operator $J: D(J) \subset X \rightarrow H$ recovers the original variable $u$ in terms of $v . P: H \rightarrow X$ is a linear operator which acts as a sort of projection into the state space $X$. For the details, see $[24,4]$.

The core of this approach is the existence of the linear operator $J$ which maps the solution of the state equation (1.2) into the (unique) solution of (1.1). (More details on this will be provided later.) Therefore, once we have proved the existence of a solution of the state equation, with a little effort we also get the existence of a solution of the Volterra equation (1.1).

There is a huge literature concerning integro-differential equations; see, for instance, the monograph [32]. Other semigroup approaches have been developed, too, among them the history function approach, which also appears in the study of stochastic equations (see, for instance, [3]). In general, stochastically perturbed problems have been treated with the aid of the resolvent operator; compare, for instance, the paper [8]. However, this approach misses the semigroup property and cannot be applied here.

We notice that (1.2) is a stochastic equation with unbounded coefficients. Similar equations are present in the literature, even though they are not completely classical. Usually they appear in the study of the Zakai equation, studied also in connection with optimal control (see, for instance, $[27,30]$ ), or stochastic equations with boundary noise (compare [33] or [11, section 11]). The same approach is used in the paper [13], where a finite horizon optimal control problem for the stochastic heat equation on a bounded real interval with Neumann boundary conditions is reformulated as a stochastic evolution equation with an unbounded operator in the diffusion term. In $[18,26]$ the authors deal with the heat equation on a halfline with boundary control and Dirichlet boundary noise. They reformulate the problem as a stochastic evolution equation in a suitable weighted $L^{2}$ space. However, in these cases, they are concerned with linear unbounded operators acting on the control or on the solution.

Our equation does not enter in this setting and deserves a specific treatment, which extends the finite dimensional case studied in [5]. In particular, since the 
forcing term $F$ is only locally Lipschitz continuous in the second component, we shall employ an approximation procedure in order to get the existence of the solution.

In section 3 we prove that for every admissible control $\gamma$ there exists a unique solution $v$ to problem (1.2). Hence in section 4 we show that the process

$$
u(t)= \begin{cases}u_{0}(t), & t \leq 0, \\ J v(t), & t \in[0, T],\end{cases}
$$

is the unique solution of the stochastic Volterra equation (1.1).

In general, optimal control problems arising in the context of analytic dynamics present more regularity than those in the case of general dynamics; this observation supports our choice of the construction of a state space setting in order to solve the Volterra equation (1.1). We translate the control problem in the state setting: our purpose will be to minimize over all admissible controls the cost functional

$$
\mathbb{J}\left(v_{0}, \gamma\right)=\mathbb{E} \int_{0}^{T} l(t, J(v(t)), \gamma(t)) \mathrm{d} t+\mathbb{E}[\phi(J(v(T)))] .
$$

In order to solve the control problem we use the forward-backward system approach. In comparison with the existing literature, we must emphasize that we consider a degenerate control problem (since nothing is assumed on the image of $g$ ) essentially in the setting assumed in [20] but, differently from them, we treat the infinite dimensional case. Moreover, the coefficients in the cost functional are unbounded operators in the state variable $v(t)$ and admit quadratic growth in the state and control.

We define in a classical way the Hamiltonian function associated to the above problem:

$$
\psi(t, v, z)=\inf \{l(t, J(v), \gamma)+z \cdot r(t, J(v), \gamma): \gamma \in \Xi\} .
$$

We take an arbitrary, complete probability space $\left(\Omega, \mathcal{F}, \mathbb{P}^{\circ}\right)$ and a Wiener process $W^{\circ}$ in $U$ with respect to $\mathbb{P}^{\circ}$. We denote by $\left(\mathcal{F}_{t}^{\circ}\right)$ the associated Brownian filtration, i.e., the filtration generated by $W^{\circ}$ and augmented by the $\mathbb{P}^{\circ}$-null sets of $\mathcal{F} ;\left(\mathcal{F}_{t}^{\circ}\right)$ satisfies the usual conditions. We introduce the forward equation

$$
\begin{aligned}
\mathrm{d} v(t) & =B v(t) \mathrm{d} t+(I-B) P G(t, v(t)) \mathrm{d} W_{t}^{\circ}, \\
v(0) & =v_{0},
\end{aligned}
$$

whose solution is a continuous $\left(\mathcal{F}_{t}^{\circ}\right)$-adapted process, which exists and is unique by the results in section 3 . Next we consider the associated backward equation of parameters $(\psi, T, \phi(J(v(t))))$

$$
\begin{aligned}
d Y_{t} & =-\psi\left(t, v(t), Z_{t}\right) \mathrm{d} t+Z_{t} \mathrm{~d} W_{t}^{\circ}, \quad t \in[0, T], \\
Y_{T} & =\phi(J(v(t))) .
\end{aligned}
$$

The solution of (1.5) exists in the sense specified in Proposition 5.5: there exist Borel measurable functions $\theta$ and $\zeta$ with values in $\mathbb{R}$ and $U^{*}$, respectively, such that the processes $Y, Z$ defined by

$$
Y_{t}=\theta(t, v(t)), \quad Z_{t}=\zeta(t, v(t))
$$

$(v$ denotes the solution of (1.4)) satisfy

$$
\mathbb{E}^{\circ} \sup _{t \in[0, T]}\left|Y_{t}\right|^{2}<\infty, \quad \mathbb{E}^{\circ} \int_{0}^{T}\left|Z_{t}\right|^{2} \mathrm{~d} t<\infty .
$$


Finally, this solution is the maximal solution among all the solutions $\left(Y^{\prime}, Z^{\prime}\right)$ of $(1.5)$ satisfying

$$
\mathbb{E}^{\circ} \sup _{t \in[0, T]}\left|Y_{t}^{\prime}\right|^{2}<\infty .
$$

The difficulty here is that the Hamiltonian corresponding to the control problem has quadratic growth in the gradient, and consequently the associated backward stochastic differential equation (BSDE) has quadratic growth in the $Z$ variable. Wellposedness for this class of BSDEs has been proved in [25] in the case of bounded terminal value. Since we allow for unbounded terminal cost, to treat such equations we have to suitably modify the techniques introduced in [6] and already used in [20]. This point requires particular attention, because we cannot use directly a monotone stability result (see Proposition 2.4 in [25]), well known in the finite dimensional framework. We notice that for such BSDEs no general uniqueness results are known: we replace uniqueness with the selection of a maximal solution.

Our main result, Corollary 5.8, proves that the optimal feedback control exists and the optimal cost is given by the value $Y_{0}$ of the maximal solution $(Y, Z)$ of the BSDE (1.5) with quadratic growth and unbounded terminal value.

Finally we address the problem of finding a weak solution to the so-called closed loop equation. If we assume that the infimum in (1.3) is attained, we can prove that there exists a measurable function $\mu$ of $t, v, z$ such that

$$
\psi(t, v, z)=l(t, J(v), \mu(t, v, z))+z \cdot r(t, J(v), \mu(t, v, z)) .
$$

We define $\bar{\gamma}(t, v)=\mu(t, v, \zeta(t, v))$, where $\zeta$ is defined above. The closed loop equation is

$$
\begin{aligned}
\mathrm{d} v(t) & =[B v(t)+(I-B) P F(t, v(t), \bar{\gamma}(t, v(t)))] \mathrm{d} t+(I-B) P G(t, v(t)) \mathrm{d} W(t), \\
v(0) & =v_{0} .
\end{aligned}
$$

First, we prove that there exists a weak solution of the closed loop equation, satisfying in addition

$$
\mathbb{E} \int_{0}^{T}|\bar{\gamma}(t, v(t))|^{2} \mathrm{~d} t<\infty .
$$

Moreover, in Corollary 5.11 we construct an optimal feedback control: if $v$ is a solution to (1.6) and we set $\gamma_{s}^{*}=\bar{\gamma}(s, v(s))$, then $\mathbb{J}\left(v_{0}, \gamma^{*}\right)=\theta\left(0, v_{0}\right)$, and consequently $v$ is an optimal state, $\gamma^{*}(s)$ is an optimal control, and $\bar{\gamma}$ is an optimal feedback.

2. Volterra equations. Integro-partial-differential equations are widely used in literature for modeling various technological applications related to the study of creep of metal, plastic materials, concrete, rock, and other bodies. In particular, we recall the applications in viscoelasticity theory (see, for instance, [32, section 5] for several examples on how the state of a mechanical system depends on the whole history of actions that were performed on it) and fractional diffusion-wave equations. This last kind of equation is modeled after

$$
\frac{\partial^{\alpha}}{\partial t^{\alpha}} u(t, x)=b \frac{\partial^{2}}{\partial x^{2}} u(t, x),
$$

where $\alpha$ is a parameter describing the order of the fractional derivative, that is taken in the sense of the Caputo fractional derivative. Although theoretically $\alpha$ can be any 
number, we consider here only the case $0<\alpha<1$; notice that for $\alpha=1$ the above equation represents the standard diffusion equation. The fractional derivative of $u$ of order $\alpha$ is

$$
\frac{\partial^{\alpha}}{\partial t^{\alpha}} u(t)=\frac{\mathrm{d}}{\mathrm{d} t} \int_{0}^{t} g_{1-\alpha}(t-s) u(s) \mathrm{d} s,
$$

where $g_{\beta}$ is the fractional derivative kernel $g_{\beta}(t)=\frac{1}{\Gamma(\beta)} t^{\beta-1}$. This kernel is continuous, locally integrable on $\mathbb{R}_{+}$, and completely monotone. There has been a growing interest in investigating this equation, for various reasons; we quote from [1], among others, "modeling of anomalous diffusive and subdiffusive systems" and "description of fractional random walks."

2.1. A motivating example. The next example shows an application of (1.1) to the theory of rigid conductors of heat composed of materials with memory.

Let $\mathcal{O} \subset \mathbb{R}^{n}$ be a fixed bounded domain with Lipschitz boundary occupied by a rigid heat conductor. The linearized theory of heat conduction in rigid material with memory is based on the classical works of [9] and [29]. If we consider only small variations of the absolute temperature and temperature gradient from equilibrium reference values, we may suppose that the internal energy $e: \mathbb{R} \times \mathcal{O} \rightarrow \mathbb{R}$ and the heat flux vector $q(t, x): \mathbb{R} \times \mathcal{O} \rightarrow \mathbb{R}^{n}$ are described by the following constitutive equations:

$$
\begin{aligned}
& e(t, x)=e_{0}+\int_{-\infty}^{t} c(t-s) \theta(s, x) \mathrm{d} s, \\
& q(t, x)=-k_{0} \nabla \theta(t, x),
\end{aligned}
$$

where $\theta: \mathbb{R} \times \mathcal{O} \rightarrow \mathbb{R}$ is the temperature variation field relative to the equilibrium reference value. In this model, we assume that the specific heat has a term of fading memory type described by the energy relaxation function $c: \mathbb{R}_{+} \rightarrow \mathbb{R}$, which we assume to be locally integrable in $\mathbb{R}_{+}$. This will allow us to consider kernels as $c(t)=t^{\alpha-1} e^{-\omega t}, \alpha \in(0,1), \omega>0$. Equation (2.1) above is simply Fourier's law. The constants $e_{0}$ and $k_{0}$ denote the internal energy at equilibrium and the instantaneous conductivity, respectively.

We consider the energy balance equation for a rigid stationary heat conductor

$$
\frac{\partial}{\partial t} e+\nabla \cdot q=h
$$

and we assume that a nonlinear temperature dependent, stochastically perturbed heat source $h$ is involved, namely,

$$
h(t, x)=\left[\sum_{j=1}^{d} \sigma_{j}(\theta(t, x)) \gamma_{j}(t)+\sum_{j=1}^{d} \sigma_{j}(\theta(t, x)) d \beta_{j}(t)\right] .
$$

Here $\sigma_{j}, j=1, \ldots, d$, are given functions $\mathbb{R} \rightarrow \mathbb{R}$, Lipschitz continuous, and bounded. $\gamma=\left(\gamma_{1}, \ldots, \gamma_{d}\right)$ acts as the control on the system. Moreover $\beta_{j}, j=1, \ldots, d$, are independent real Wiener processes.

In this framework, subject, for instance, to Dirichlet boundary conditions, the problem reads as follows:

$$
\frac{\partial}{\partial t} \int_{-\infty}^{t} c(t-s) \theta(s, x) \mathrm{d} s-k_{0} \Delta \theta(t, x)=h(t, x),
$$

Copyright (c) by SIAM. Unauthorized reproduction of this article is prohibited. 
which is a nonlinear dynamical system. In other terms, $\theta$ is not a Markov process, since its future development depends on the whole history. With the choice $c(t)=$ $g_{\beta}(t)=\frac{1}{\Gamma[1-\beta]} t^{-\beta}$, the above equation reads $D^{\beta} \theta(t)=k_{0} \Delta \theta(t)+h(t)$, where $D^{\beta}$ is the fractional derivative of order $\beta$; compare, for instance, [32].

We can consider the following quadratic cost functional:

$$
\mathbb{J}\left(\theta_{0}, \gamma\right)=\mathbb{E} \int_{0}^{T}\left[\frac{1}{2}|\gamma(t)|_{\mathbb{R}^{d}}^{2}+\int_{\mathcal{O}}|\theta(t, x)|^{2} \mathrm{~d} x\right] \mathrm{d} t+\mathbb{E}|\theta(T, x)|^{2} .
$$

The control problem requires us to minimize the functional $\mathbb{J}$ over all admissible control processes $\gamma \in L^{2}\left(\Omega \times[0, T] ; \mathbb{R}^{d}\right)$.

The above example will be solved in section 5.3, where we shall see how it fits into our setting and which kind of solution this problem admits.

2.2. Main assumptions and remarks. The convolution kernel $a:(0,+\infty) \rightarrow$ $\mathbb{R}$ in (1.1) is completely monotone. This means that $a:(0,+\infty) \rightarrow \mathbb{R}$ is a continuous, monotone decreasing function, infinitely often derivable, such that

$$
(-1)^{n} \frac{\mathrm{d}^{n}}{\mathrm{~d} t^{n}} a(t) \geq 0, \quad t>0, n=0,1,2, \ldots .
$$

We assume that $a$ is locally integrable and $a(0+)=+\infty$ and $\int_{0}^{1} a(s) \mathrm{d} s<+\infty$. We shall control the level of singularity of $a$. The following construction is rather technical: compare [4] for further details. First, for the completely monotone kernel $a$ we define the numbers

$$
\begin{aligned}
& \alpha(a):=\sup \left\{\rho \in(0,1) \mid \int_{c}^{+\infty} s^{\rho-2} \frac{1}{\hat{a}(s)} \mathrm{d} s<+\infty\right\}, \\
& \delta(a):=\inf \left\{\rho \in(0,1) \mid \int_{c}^{+\infty} s^{-\rho} \hat{a}(s) \mathrm{d} s<+\infty\right\}
\end{aligned}
$$

for some $c>0$. Then we require

$$
\alpha(a)>\frac{1}{2}
$$

The definitions of $\alpha(a)$ and $\delta(a)$ are independent of the choice of the number $c>0$. It is always true that $\alpha(a) \leq \delta(a)$, but there are examples of (rather artificial) completely monotone kernels $a$ with $\alpha(a)<\delta(a)$.

These quantities are related to the power of the singularity of the kernel at $0+$, as the following example shows.

Remark 2.1. Let $a(t)=e^{-\omega t} t^{\alpha-1}$, where $\omega>0$, be the kernel in subsection 2.1. This kernel is completely monotone with Laplace transform $\hat{a}(s)=\Gamma[\alpha](\omega+s)^{-\alpha}$; an easy computation shows that $\alpha(a)=1-\alpha$, and hence we satisfy Assumption (2.2) whenever we take $\alpha \in\left(0, \frac{1}{2}\right)$.

We proceed with the main assumptions on the coefficients of the Volterra equation (1.1). Here, we focus on the leading operator $A$ and the space $X$.

Assumption 2.2.

(i) $A: D(A) \subset H \rightarrow H$ is a sectorial operator in $H$. Thus $A$ generates an analytic semigroup $e^{t A}$. Interpolation and extrapolation spaces $H_{\eta}$ of $H$ will always be constructed with respect to $A$.

Copyright $@$ by SIAM. Unauthorized reproduction of this article is prohibited. 
(ii) The initial condition $u_{0}(t)$ belongs to the space $\tilde{X}_{0}$ of admissible initial conditions,

$$
\begin{aligned}
\tilde{X}_{0}=\left\{u: \mathbb{R}_{-} \rightarrow H, \text { there exist } M>0 \text { and } \omega>0\right. \\
\\
\text { such that } \left.|u(t)| \leq M e^{-\omega t}\right\},
\end{aligned}
$$

and it satisfies further the following assumption:

(a) $u_{0}(0)$ belongs to $H_{\epsilon}$ for some $\epsilon \in\left(0, \frac{1}{2}\right)$ and there exist $M_{2}>0$ and $\tau>0$ such that $\left|u_{0}(t)-u_{0}(0)\right| \leq M|t|$ for all $\left.t \in[-\tau, 0]\right)$.

2.3. The state space setting. It is known that we can associate to any completely monotone kernel $a$, by means of Bernstein's theorem [32, p. 90], a positive measure $\nu$ on $[0,+\infty)$ such that

$$
a(t)=\int_{[0,+\infty)} e^{-\kappa t} \nu(\mathrm{d} \kappa) .
$$

From the required singularity of $a$ at $0+$ we obtain that $\nu([0,+\infty))=a(0+)=+\infty$, while for $s>0$ the Laplace transform $\hat{a}$ of $a$ verifies

$$
\hat{a}(s)=\int_{[0,+\infty)} \frac{1}{s+\kappa} \nu(\mathrm{d} \kappa)<+\infty .
$$

Under the assumption of complete monotonicity of the kernel, a semigroup approach to a type of abstract integro-differential equation encountered in linear viscoelasticity was introduced in [14]. We recall the extension given in [4] to the case of Hilbert space valued equations. We start for simplicity with the equation

$$
\begin{aligned}
\frac{d}{d t} \int_{-\infty}^{t} a(t-s) u(s) \mathrm{d} s & =A u(t)+f(t), & t \in[0, T], \\
u(t) & =u_{0}(t), & t \leq 0 .
\end{aligned}
$$

The starting point is the following identity, which follows by Bernstein's theorem,

$$
\int_{-\infty}^{t} a(t-s) u(s) \mathrm{d} s=\int_{-\infty}^{t} \int_{[0,+\infty)} e^{-\kappa(t-s)} \nu(\mathrm{d} \kappa) u(s) \mathrm{d} s=\int_{[0,+\infty)} v(t, \kappa) \nu(\mathrm{d} \kappa),
$$

where we introduce the state variable

$$
v(t, \kappa)=\int_{-\infty}^{t} e^{-\kappa(t-s)} u(s) \mathrm{d} s
$$

Formal differentiation yields

$$
\frac{\partial}{\partial t} v(t, \kappa)=-\kappa v(t, \kappa)+u(t)
$$

while the integral equation (2.3) can be rewritten as

$$
\int_{[0,+\infty)}(-\kappa v(t, \kappa)+u(t)) \nu(\mathrm{d} \kappa)=A u(t)+f(t) .
$$

Now, the idea is to use (2.4) as the state equation with $B v=-\kappa v(\kappa)+u$, while (2.5) enters in the definition of the domain of $B$. 
In our setting, the function $v(t, \cdot)$ will be considered the state of the system, contained in the state space $X$ that consists of all Borel measurable functions $\tilde{x}$ : $[0,+\infty) \rightarrow H$ such that the seminorm

$$
\|\tilde{x}\|_{X}^{2}:=\int_{[0,+\infty)}(\kappa+1)|\tilde{x}(\kappa)|_{H}^{2} \nu(\mathrm{d} \kappa)
$$

is finite. We shall identify the classes $\tilde{x}$ with respect to equality almost everywhere in $\nu$.

Let us consider the initial condition. On the space $\tilde{X}_{0}$ introduced in Assumption 2.2 we define a positive inner product

$$
\langle u, v\rangle_{\tilde{X}}=\iint\left[a(t+s)-a^{\prime}(t+s)\right]\langle u(-s), v(-t)\rangle_{H} \mathrm{~d} s \mathrm{~d} t
$$

then, setting $\tilde{N}_{0}=\left\{u \in \tilde{X}_{0}:\langle u, u\rangle_{\tilde{X}}=0\right\},\langle\cdot, \cdot\rangle_{\tilde{X}}$ is a scalar product on $\tilde{X}_{0} / \tilde{N}_{0}$, we define $\tilde{X}$ the completition of this space with respect to $\langle\cdot, \cdot\rangle_{\tilde{X}}$. We let the operator $Q: \tilde{X} \rightarrow X$ be given by

$$
v(0, \kappa)=Q u_{0}(\kappa)=\int_{-\infty}^{0} e^{\kappa s} u_{0}(s) \mathrm{d} s .
$$

It has been proved in [4, Proposition 2.5] that the operator $Q$ is an isometric isomorphism between $\tilde{X}$ and $X$. This operator maps the initial value of the stochastic Volterra equation in the initial value of the abstract state equation. Different initial conditions of the Volterra equation generate different initial conditions of the state equation.

Condition (a) in Assumption 2.2(ii) is necessary in order to have greater regularity on the initial value of the state equation. In fact, in this case [4, Proposition 2.20] shows that $Q u_{0}$ belongs to $X_{\eta}$ for $\eta \in\left(0, \frac{1}{2}\right)$.

Remark 2.3. We stress that under our assumptions we are able to treat, for instance, initial conditions for the Volterra equation of the form

$$
u_{0}(t)= \begin{cases}0, & (-\infty,-\delta), \\ \bar{u}, & {[-\delta, 0],}\end{cases}
$$

provided $\bar{u}$ has a suitable regularity.

We quote from [4] the main result concerning the state space setting for stochastic Volterra equations in infinite dimensions.

TheOrem 2.4 (state space setting). Let $A, a, \alpha(a)$ be given above; choose numbers $\eta \in(0,1), \theta \in(0,1)$ such that

$$
\eta>\frac{1}{2}(1-\alpha(a)), \quad \theta<\frac{1}{2}(1+\alpha(a)), \quad \theta-\eta>\frac{1}{2} .
$$

Then there exist

(1) a separable Hilbert space $X$ and an isometric isomorphism $Q: \tilde{X} \rightarrow X$,

(2) a densely defined sectorial operator $B: D(B) \subset X \rightarrow X$ generating an analytic semigroup $e^{t B}$ with growth bound $\omega_{0}$,

(3) its real interpolation spaces $X_{\rho}=(X, D(B))_{(\rho, 2)}$ with their norms $\|\cdot\|_{\rho}$,

(4) linear operators $P: H \rightarrow X_{\theta}, J: X_{\eta} \rightarrow H$ 
such that the following holds: For each $v_{0} \in X$, the problem (2.3) is equivalent to the evolution equation

$$
\begin{aligned}
v^{\prime}(t) & =B v(t)+(I-B) \operatorname{Pf}(t), \\
v(0) & =v_{0}
\end{aligned}
$$

in the sense that if $u_{0} \in \tilde{X}_{0}$ and $v\left(t ; v_{0}\right)$ is the weak solution to problem (2.6) with $v_{0}=Q u_{0}$, then $u\left(t ; u_{0}\right)=J v\left(t ; v_{0}\right)$ is the unique weak solution to problem (2.3).

It is remarkable that $B$ generates an analytic semigroup, since in this case we have at our disposal a powerful theory of optimal regularity results. In particular, besides the interpolation spaces $X_{\theta}$ introduced in Theorem 2.4, we may construct the extrapolation space $X_{-1}$, i.e., a Sobolev space of negative order associated to $e^{t B}$.

Assume for simplicity that $B$ is of negative type. (Otherwise, one may consider $B-\omega_{0}$ instead of $B$ in the following discussion.) The semigroup $e^{t B}$ extends to $X_{-1}$ and the generator of this extension, that we denote $B_{-1}$, is the unique continuous extension of $B$ to an isometry between $X$ and $X_{-1}$. See, for instance, [15, Definition 5.4] for further details.

Remark 2.5. In what follows, we shall always denote the operator with the letter $B$, even in the case where formally $B_{-1}$ should be used instead. This should cause no confusion, due to the similarity of the operators.

2.4. Preliminary results on existence and uniqueness for the linear, nonhomogeneous Volterra equation. As a preliminary step for what follows, we state some results for the linear, nonhomogeneous Volterra equation, which we will use mainly in section 4 .

The first is a simple, yet important, uniqueness result for (a special case of) the Volterra equation.

Proposition 2.6. The linear equation

$$
\begin{aligned}
\frac{d}{d t} \int_{-\infty}^{t} a(t-s) u(s) \mathrm{d} s & =A u(t), \quad t \in[0, T], \\
u(t) & =0, \quad t \leq 0 .
\end{aligned}
$$

has a unique solution $u \equiv 0$.

Proof. Let us take the Laplace transform in both sides of the linear equation (2.7); we obtain

$$
\lambda \hat{a}(\lambda) \hat{u}(\lambda)=A \hat{u}(\lambda), \quad \Re \lambda \geq 0, \lambda \neq 0
$$

therefore, $\hat{u}(\lambda)=R(\lambda \hat{a}(\lambda), A) 0$.

Let $\lambda=x+i y$; recall that by Bernstein's theorem, $\nu$ is the unique measure associated with the kernel $a$. Using [24, Lemma 1.1.7] we have

$$
\lambda \hat{a}(\lambda)=\int_{[0, \infty)} \frac{(x+i y) \kappa+\left(x^{2}+y^{2}\right)}{(\kappa+x)^{2}+y^{2}} \nu(\mathrm{d} \kappa),
$$

hence $\Re(\lambda \hat{a}(\lambda)) \geq 0$ for all $\Re \lambda \geq 0, \lambda \neq 0$, which means that $\lambda \hat{a}(\lambda) \in \rho(A)$ and

$$
\hat{u}(\lambda)=0, \quad \Re \lambda \geq 0, \lambda \neq 0 .
$$

The complex inversion formula for the Laplace transform therefore leads to

$$
u(t)=0 \quad \text { for a.a. } t \geq 0
$$

as claimed. 
Now we deal with existence and uniqueness of the stochastic Volterra equation with nonhomogeneous terms. To this end we extend the result in [4, Theorem 3.7] where the case $f(t) \equiv 0$ is treated.

Proposition 2.7. In our assumptions, let $v_{0} \in X_{\eta}$ for some $\frac{1-\alpha(a)}{2}<\eta<$ $\frac{1}{2} \alpha(a)$. Given the process

$$
v(t)=e^{t B} v_{0}+\int_{0}^{t} e^{(t-s) B}(I-B) P f(s) \mathrm{d} s+\int_{0}^{t} e^{(t-s) B}(I-B) P g(s) \mathrm{d} W(s)
$$

we define the process

$$
u(t)= \begin{cases}J v(t), & t \geq 0 \\ u_{0}(t), & t \leq 0\end{cases}
$$

Then $u(t)$ is a weak solution to problem

$$
\begin{aligned}
\frac{d}{d t} \int_{-\infty}^{t} a(t-s) u(s) \mathrm{d} s & =A u(t)+f(t)+g(t) \dot{W}(t), \quad t \in[0, T], \\
u(t) & =u_{0}(t), \quad t \leq 0 .
\end{aligned}
$$

Before to prove the result we recall the definition of weak solution for stochastic Volterra equations.

Definition 2.8. According to [12, section 6.1], a process $u=\{u(t), t \in[0, T]\}$ is a weak solution to (2.9) if $u$ is an $H$-valued predictable process with

$$
\mathbb{E} \int_{0}^{T}|u(s)|^{2} \mathrm{~d} s<+\infty
$$

and the identity

$$
\begin{aligned}
\int_{-\infty}^{t} a(t-s)\langle u(s), \zeta\rangle_{H} \mathrm{~d} s= & \langle\bar{u}, \zeta\rangle_{H}+\int_{0}^{t}\left\langle u(s), A^{\star} \zeta\right\rangle_{H} \mathrm{~d} s \\
& +\int_{0}^{t}\langle f(s), \zeta\rangle_{H} \mathrm{~d} s+\int_{0}^{t}\langle g(s) \mathrm{d} W(s), \zeta\rangle_{H}
\end{aligned}
$$

holds $\mathbb{P}$-a.s. for arbitrary $t \in[0, T]$ and $\zeta \in D\left(A^{\star}\right)$, where $A^{\star}$ is the adjoint of the operator $A$ and

$$
\bar{u}=\int_{-\infty}^{0} a(-s) u_{0}(s) \mathrm{d} s .
$$

Proof of Proposition 2.7. The operator $J$, introduced in Theorem 2.4, acts as a projection from the state space $X$ to $H . J$ is a linear unbounded operator with domain $D(J)$ strictly contained in $X$. In [4, Lemma 3.11] it was proved that this domain contains an interpolation space $X_{\eta}$ and, with a slight abuse of notation, we shall denote again with $J$ the restriction of the projection operator from $X_{\eta}$ to $H$.

The mapping $P: H \rightarrow X$ introduced in Theorem 2.4 is defined by

$$
(P f)(\kappa)=\frac{1}{1+\kappa} R(\hat{a}(1), A) f .
$$

Copyright (c) by SIAM. Unauthorized reproduction of this article is prohibited. 
The above explicit formula will be useful in the rest of this proof. It is a matter of computation to show that $P f$ actually belongs to $X_{\theta}$ for $\theta<\frac{1}{2}(1+\alpha(a))$ as stated in that theorem.

As in the discussion preceding Remark 2.5, we define $B_{\eta}$ to be the restriction of $B$ as an operator $B_{\eta}: X_{\eta+1} \rightarrow X_{\eta}$. As usual, $B^{\star}$ is the adjoint of $B$.

For fixed $\zeta \in D\left(A^{\star}\right)$, we define the vector $\xi \in X$ by $\xi(\kappa)=\frac{1}{1+\kappa} \zeta$. We claim that $\xi \in D\left(B_{\eta}^{*}\right)$. Actually, for $x \in X_{\eta+1}$ we have

$$
\begin{aligned}
\left\langle\xi, B_{\eta} x\right\rangle_{X} & =\int_{[0, \infty)}(\kappa+1)\left\langle\frac{1}{1+\kappa} \zeta,(-\kappa x(\kappa)+J x)\right\rangle_{H} \nu(\mathrm{d} \kappa) \\
& =\left\langle\zeta, \int_{[0, \infty)}(-\kappa x(\kappa)+J x) \nu(\mathrm{d} \kappa)\right\rangle_{H}=\langle\zeta, A J x\rangle_{H}=\left\langle A^{\star} \zeta, J x\right\rangle .
\end{aligned}
$$

Moreover, by [4, Lemma 3.10]

$$
\Psi(t)=(I-B) P g(t) \in L_{\mathcal{F}}^{2}\left(\Omega \times(0, T) ; L_{2}\left(U, X_{\theta-1}\right)\right) \quad \text { for any } \theta<\frac{\alpha(a)+1}{2} .
$$

We have that the process $v$, defined in (2.8), solves the following equation in weak form:

$$
\begin{aligned}
\langle v(t), \xi\rangle_{X}= & \left\langle v_{0}, \xi\right\rangle_{X}+\int_{0}^{t}\left\langle B_{\eta}^{\star} \xi, v(s)\right\rangle \mathrm{d} s+\int_{0}^{t}\langle\xi,(I-B) P f(s)\rangle_{X} \mathrm{~d} s \\
& +\int_{0}^{t}\langle\xi, \Psi(s) \mathrm{d} W(s)\rangle_{X} .
\end{aligned}
$$

We use the above representation in order to prove that $u$ is a weak solution of (2.9).

Let us consider separately the several terms. The initial condition yields

$$
\begin{aligned}
\left\langle v_{0}, \xi\right\rangle_{X} & =\int_{[0, \infty)}\langle v(0, \kappa), \zeta\rangle_{H} \nu(\mathrm{d} \kappa) \\
& =\left\langle\int_{[0, \infty)} \int_{[0, \infty)} e^{-\kappa s} u_{0}(-s) \mathrm{d} s \nu(\mathrm{d} \kappa), \zeta\right\rangle_{H}=\langle\bar{u}, \zeta\rangle_{H}
\end{aligned}
$$

where $\bar{u}$ is defined in (2.11); next, the second term can be evaluated using equality (2.12),

$$
\int_{0}^{t}\left\langle B_{\eta}^{\star} \xi, v(s)\right\rangle_{X} \mathrm{~d} s=\int_{0}^{t}\left\langle A^{\star} \zeta, J v(s)\right\rangle_{H} \mathrm{~d} s=\int_{0}^{t}\left\langle A^{\star} \zeta, u(s)\right\rangle_{H} \mathrm{~d} s .
$$

The third one leads to

$$
\int_{0}^{t}\langle\xi,(I-B) \operatorname{Pf}(s)\rangle_{X} \mathrm{~d} s=\int_{0}^{t}\langle\xi, P f(s)\rangle_{X} \mathrm{~d} s-\int_{0}^{t}\left\langle B^{\star} \xi, P f(s)\right\rangle_{X} \mathrm{~d} s
$$

and we consider the two terms separately:

$$
\begin{aligned}
\langle\xi, P f(s)\rangle_{X} & =\int_{[0, \infty)}(\kappa+1)\left\langle\frac{1}{\kappa+1} \zeta, \frac{1}{\kappa+1} R(\hat{a}(1), A) f(s)\right\rangle_{H} \nu(\mathrm{d} \kappa) \\
& =\langle\zeta, \hat{a}(1) R(\hat{a}(1), A) f(s)\rangle_{H}
\end{aligned}
$$

Copyright (C) by SIAM. Unauthorized reproduction of this article is prohibited. 
and

$$
\left\langle B^{\star} \xi, \operatorname{Pf}(s)\right\rangle_{X}=\langle\zeta, \operatorname{AJPf}(s)\rangle_{H}=\langle\zeta, A R(\hat{a}(1), A) f(s)\rangle_{H}
$$

hence

$$
\begin{aligned}
\int_{0}^{t}\langle\xi,(I-B) P f(s)\rangle_{X} \mathrm{~d} s= & \int_{0}^{t}\langle\zeta, \hat{a}(1) R(\hat{a}(1), A) f(s)\rangle_{H} \mathrm{~d} s \\
& -\int_{0}^{t}\langle\zeta, A R(\hat{a}(1), A) f(s)\rangle_{H} \mathrm{~d} s=\int_{0}^{t}\langle\zeta, f(s)\rangle_{H} \mathrm{~d} s .
\end{aligned}
$$

We finally turn to the stochastic integral. For an orthonormal basis $\left\{e_{j}\right\}$ in $U$ we let $\beta_{j}(t)=\left\langle W(t), e_{j}\right\rangle_{U}$ and $\Psi_{j}(t)=(I-B) P g(s) \cdot e_{j}$; then

$$
\begin{aligned}
\int_{0}^{t}\langle\xi, \Psi(s) \mathrm{d} W(s)\rangle_{X} & =\sum_{j=1}^{\infty} \int_{0}^{t}\left\langle\xi, \Psi_{j}(s)\right\rangle_{X} \mathrm{~d} \beta_{j}(s) \\
& =\sum_{j=1}^{\infty} \int_{0}^{t}\left\langle\xi,(I-B) P g(s) \cdot e_{j}\right\rangle_{X} \mathrm{~d} \beta_{j}(s) .
\end{aligned}
$$

This quantity can be treated as we have done for (2.13) and we obtain

$$
\begin{aligned}
\int_{0}^{t}\langle\xi, \Psi(s) \mathrm{d} W(s)\rangle_{X} & =\sum_{j=1}^{\infty} \int_{0}^{t}\left\langle\zeta,(\hat{a}(1)-A) R(\hat{a}(1), A) g(s) \cdot e_{j}\right\rangle_{H} \mathrm{~d} \beta_{j}(s) \\
& =\sum_{j=1}^{\infty} \int_{0}^{t}\left\langle\zeta, g(s) \cdot e_{j}\right\rangle_{H} \mathrm{~d} \beta_{j}(s)=\int_{0}^{t}\langle\zeta, g(s) \mathrm{d} W(s)\rangle_{H} .
\end{aligned}
$$

We have proved so far that

$$
\begin{aligned}
\langle v(t), \xi\rangle_{X}= & \langle\bar{u}, \zeta\rangle_{H}+\int_{0}^{t}\left\langle A^{\star} \zeta, u(s)\right\rangle_{H} \mathrm{~d} s+\int_{0}^{t}\langle\zeta, f(s)\rangle_{H} \mathrm{~d} s \\
& +\int_{0}^{t}\langle\zeta, g(s) \mathrm{d} W(s)\rangle_{H} .
\end{aligned}
$$

It only remains to prove

$$
\int_{[0, \infty)}\langle v(t, \kappa), \zeta\rangle_{H} \nu(\mathrm{d} \kappa)=\int_{-\infty}^{t}\langle a(t-s) u(s), \zeta\rangle_{H} \mathrm{~d} s
$$

If we recall the definition of $u(t)=\left\{\begin{array}{ll}J v(t), & t>0, \\ u_{0}(t), & t \leq 0,\end{array}\right.$ we obtain

$$
\int_{-\infty}^{t}\langle a(t-s) u(s), \zeta\rangle_{H} \mathrm{~d} s=\int_{-\infty}^{0}\left\langle a(t-s) u_{0}(s), \zeta\right\rangle_{H} \mathrm{~d} s+\int_{0}^{t}\langle a(t-s) J v(s), \zeta\rangle_{H} \mathrm{~d} s .
$$

We then exploit the definition of $a(t)$; the first term becomes

$$
\begin{aligned}
\int_{-\infty}^{0}\left\langle a(t-s) u_{0}(s), \zeta\right\rangle_{H} \mathrm{~d} s & =\left\langle\int_{-\infty}^{0} \int_{[0, \infty)} e^{-\kappa(t-s)} \nu(\mathrm{d} \kappa) u_{0}(s) \mathrm{d} s, \zeta\right\rangle_{H} \\
& =\left\langle\int_{[0, \infty)} e^{-\kappa t} \int_{-\infty}^{0} e^{-\kappa(-s)} u_{0}(s) \mathrm{d} s \nu(\mathrm{d} \kappa), \zeta\right\rangle_{H} \\
& =\left\langle\int_{[0, \infty)} e^{-\kappa t} v(0, \kappa) \nu(\mathrm{d} \kappa), \zeta\right\rangle_{H},
\end{aligned}
$$

Copyright $@$ by SIAM. Unauthorized reproduction of this article is prohibited. 
the second term becomes

$$
\begin{aligned}
\int_{0}^{t}\langle a(t-s) J v(s), \zeta\rangle_{H} \mathrm{~d} s & =\left\langle\int_{0}^{t} \int_{[0, \infty)} e^{-\kappa(t-s)} \nu(\mathrm{d} \kappa) J v(s) \mathrm{d} s, \zeta\right\rangle_{H} \\
& =\left\langle\int_{[0, \infty)} \int_{0}^{t} e^{-\kappa(t-s)} J v(s) \mathrm{d} s \nu(\mathrm{d} \kappa), \zeta\right\rangle_{H},
\end{aligned}
$$

and the thesis follows from the identification of the processes

$$
\begin{aligned}
v(t, \kappa)= & e^{t B} v_{0}(\kappa)+\int_{0}^{t}\left[e^{(t-s) B}(I-B) P f(s)\right](\kappa) \mathrm{d} s \\
& +\int_{0}^{t}\left[e^{(t-s) B}(I-B) P g(s)\right](\kappa) \mathrm{d} W(s) \\
\tilde{v}(t, \kappa)= & e^{-\kappa t} v_{0}(\kappa)+\int_{0}^{t} e^{-\kappa(t-s)} J v(s) \mathrm{d} s,
\end{aligned}
$$

proved in next lemma.

Lemma 2.9. In our assumptions, let $v_{0} \in X_{\eta}$ for some $\frac{1-\alpha(a)}{2}<\eta<\frac{1}{2} \alpha(a)$. Consider the processes $v$ and $\tilde{v}$ defined in (2.14). Then $\tilde{v}(t)$ is a modification of $v(t)$.

Proof. The proof follows by a Laplace transform argument which adapts to our case the ideas in [4, Proposition 3.8].

We shall denote by $\mathcal{L}$ the Laplace transform of a function:

$$
\mathcal{L}[f](s)=\int_{[0, \infty)} e^{-s t} f(t) \mathrm{d} t \text { and similarly } \mathcal{L}[g \dot{W}](s)=\int_{[0, \infty)} e^{-s t} g(t) \mathrm{d} W(t) ;
$$

if we apply the Laplace transform in the first line of (2.14) we get

$$
\begin{aligned}
\mathcal{L}[v(\cdot, \kappa)](s)= & R(s, B) v_{0}(\kappa)+[R(s, B)(I-B) P \mathcal{L}[f](s)](\kappa) \\
& +[R(s, B)(I-B) P \mathcal{L}[g \dot{W}](s)](\kappa) .
\end{aligned}
$$

Now we use the representation formulas stated in $[4,(2.14)$ and $(2.17)]$ to get

$$
\begin{aligned}
\mathcal{L}[v(\cdot, \kappa)](s)= & \frac{1}{\kappa+s}\left(v_{0}(\kappa)+R(s \hat{a}(s), A) \int_{[0, \infty)} \frac{\bar{\kappa}}{\bar{\kappa}+s} v_{0}(\bar{\kappa}) \nu(\mathrm{d} \bar{\kappa})\right) \\
& +\frac{1}{\kappa+s} R(s \hat{a}(s), A) \mathcal{L}[f](s)+\frac{1}{\kappa+s} R(s \hat{a}(s), A) \mathcal{L}[g \dot{W}](s) .
\end{aligned}
$$

Now we turn to the second process in (2.14); we obtain that the Laplace transform is

$$
\mathcal{L}[\tilde{v}](s)=\frac{1}{\kappa+s} v_{0}(\kappa)+\frac{1}{\kappa+s} \mathcal{L}[J v](s)
$$

and a direct computation shows that the above quantity is equal to (2.15).

3. Stochastic differential equations with unbounded diffusion operator. We first study (1.1) without the control. We are concerned with the equation

$$
\begin{aligned}
\frac{d}{d t} \int_{-\infty}^{t} a(t-s) u(s) \mathrm{d} s & =A u(t)+g(t, u(t)) \dot{W}(t), \quad t \in[0, T], \\
u(t) & =u_{0}(t), \quad t \leq 0 .
\end{aligned}
$$

Copyright $\odot$ by SIAM. Unauthorized reproduction of this article is prohibited. 
Besides the hypothesis in Assumption 2.2, we require the following on the coefficients of (3.1).

Assumption 3.1.

(i) The process $\left\{W_{t}, t \in[0, T]\right\}$ is a cylindrical Wiener process defined on a complete probability space $\left(\Omega, \mathcal{F},\left\{\mathcal{F}_{t}\right\}, \mathbb{P}\right)$, taking values in the Hilbert space $U$. This means that $W(t)$ is a linear mapping $W(t): U \rightarrow L^{2}(\Omega)$ such that

(a) for every $u \in U,\{W(t) \cdot u, t \geq 0\}$ is a real-valued Brownian motion, and (b) for every $u, v \in U$ and $t \geq 0, \mathbb{E}[(W(t) \cdot u)(W(t) \cdot v)]=\langle u, v\rangle_{U}$.

(ii) The mapping $g:[0, T] \times H \rightarrow L_{2}(U, H)$ (the space of Hilbert-Schmidt operators from $U$ into $H$ ) is measurable; moreover there exists a constant $C>0$ such that for every $t \in[0, T], u, u^{\prime} \in H$,

$$
\begin{aligned}
\left\|g(t, u)-g\left(t, u^{\prime}\right)\right\|_{L_{2}(U, H)} & \leq C\left|u-u^{\prime}\right|, \\
\|g(t, u)\|_{L_{2}(U, H)} & \leq C .
\end{aligned}
$$

We apply to problem (3.1) the machinery introduced in previous section; then we obtain, on the space $X$, the stochastic Cauchy problem

$$
\begin{aligned}
\mathrm{d} v(t) & =B v(t) \mathrm{d} t+(I-B) P g(t, J(v(t))) \mathrm{d} W_{t}, \\
v(s) & =v_{0}
\end{aligned}
$$

for $0 \leq s \leq t \leq T$ and initial condition $v_{0} \in X_{\eta}$. The above expression is only formal in $X_{\eta}$ since the coefficients do not belong to the state space; however, we can give a meaning to the mild form of the equation:

$$
v\left(t ; s, v_{0}\right)=e^{(t-s) B} v_{0}+\int_{s}^{t} e^{(t-\sigma) B}(I-B) P g(\sigma, J(v(\sigma))) \mathrm{d} W(\sigma) .
$$

The main result of this section is the existence for the solution of (3.2). To this end we define a mapping $\mathcal{K}$ from $L_{\mathcal{F}}^{p}\left(\Omega ; C\left([0, T] ; X_{\eta}\right)\right)$ to itself by the formula

$$
\mathcal{K}(v)(t)=e^{(t-s) B} v_{0}+\int_{s}^{t} e^{(t-\tau) B}(I-B) P g(\tau, J(v(\tau))) \mathrm{d} W(\tau), \quad t \in[s, T],
$$

and show that it is a contraction under an equivalent norm. The unique fixed point shall be the required solution.

Let us introduce the norm

$$
\|v\|_{\eta}^{p}=\mathbb{E} \sup _{t \in[0, T]} e^{-\beta p t}\|v(t)\|_{\eta}^{p}
$$

where $\beta>0$ will be chosen later. In the space $L_{\mathcal{F}}^{p}\left(\Omega ; C\left([0, T] ; X_{\eta}\right)\right)$ this norm is equivalent to the original one.

We define the mapping

$$
\Lambda(v, s)(t)=\int_{s}^{t} e^{(t-\tau) B}(I-B) \operatorname{Pg}(\tau, J(v(\tau))) \mathrm{d} W(\tau) .
$$

Our first goal is to prove that $\Lambda$ is a well-defined mapping on $L_{\mathcal{F}}^{p}\left(\Omega ; C\left([0, T] ; X_{\eta}\right)\right)$ and to give estimates on its norm. Let us stress that in the rest of the paper $\Lambda(v)$ will be defined for $t \in[0, T]$ by setting $\Lambda(v)(t)=0$ for $t<s$. 
The following result is used in the proof of Theorem 3.3. The strategy of the proof, based on the classical factorization method by Da Prato and Zabczyk, is replicated several times here.

Lemma 3.2. For every $\frac{1}{p}<\theta-\eta-\frac{1}{2}$ the operator $\Lambda$ maps $L_{\mathcal{F}}^{p}\left(\Omega ; C\left([0, T] ; X_{\eta}\right)\right)$ into itself.

Proof. Let $v \in L_{\mathcal{F}}^{p}\left(\Omega ; C\left([0, T] ; X_{\eta}\right)\right)$; for simplicity we fix the initial time $s=0$ and write $\Lambda(t)=\Lambda(v, 0)(t)$. Further, we remark that the thesis is equivalent to the statement: $t \mapsto(I-B)^{\eta} \Lambda(t) \in L_{\mathcal{F}}^{p}(\Omega ; C([0, T] ; X))$.

Step 1 . For given $\gamma \in(0,1)$ and $\eta \in(0,1)$, the following identity holds:

$$
e^{-\beta t}(I-B)^{\eta} \Lambda(t)=c_{\gamma} \int_{0}^{t} e^{-\beta(t-\tau)}(t-\tau)^{\gamma-1} e^{(t-\tau) B} y_{\eta}(\tau) \mathrm{d} \tau
$$

where $y_{\eta}$ is the process

$$
y_{\eta}(\tau)=\int_{0}^{\tau} e^{-\beta \sigma}(\tau-\sigma)^{-\gamma} e^{-\beta(\tau-\sigma)} e^{(\tau-\sigma) B}(I-B)^{\eta+1} P g(\sigma, J(v(\sigma))) \mathrm{d} W(\sigma)
$$

We shall estimate the $L^{p}(\Omega ; X)$-norm of this process:

$$
\begin{aligned}
\mathbb{E}\left|y_{\eta}(\tau)\right|^{p}=\mathbb{E} \mid & \int_{0}^{\tau} e^{-\beta \sigma}(\tau-\sigma)^{-\gamma} \\
& \left.e^{-\beta(\tau-\sigma)} e^{(\tau-\sigma) B}(I-B)^{\eta+1} \operatorname{Pg}(\sigma, J(v(\sigma))) \mathrm{d} W(\sigma)\right|^{p} .
\end{aligned}
$$

Proceeding as in [12, Lemma 7.2] this leads to

$\mathbb{E}\left|y_{\eta}(\tau)\right|^{p}$

$\leq C \mathbb{E}\left[\int_{0}^{\tau}\left\|e^{-\beta \sigma} e^{(\tau-\sigma) B}(I-B)^{\eta+1} \operatorname{Pg}(\sigma, J((\sigma)))(\tau-\sigma)^{-\gamma} e^{-\beta(\tau-\sigma)}\right\|_{L_{2}(U, X)}^{2} \mathrm{~d} \sigma\right]^{p / 2}$.

Since the semigroup $e^{t B}$ is analytic, $P$ maps $H$ into $X_{\theta}$ for arbitrary $\theta<\frac{1+\alpha(a)}{2}$, and $g$ takes values in $L_{2}(U, H)$, the following estimate holds:

$$
\left\|e^{(\tau-\sigma) B}(I-B)^{\eta+1} P g(\sigma, J(v(\sigma)))\right\|_{L_{2}(U, X)} \leq C(\tau-\sigma)^{\theta-1-\eta}\|g(\sigma, J(v(\sigma)))\|_{L_{2}(U, H)}
$$

and the process $y_{\eta}$ is estimated by

$$
\begin{aligned}
\mathbb{E}\left|y_{\eta}(\tau)\right|^{p} \leq C \mathbb{E} & \left(\int_{0}^{\tau} e^{-2 \beta \sigma}\|g(\sigma, J(v(\sigma)))\|_{L_{2}(U, H)}^{2}\right. \\
& \left.e^{-2 \beta(\tau-\sigma)}(\tau-\sigma)^{-2(\gamma+1+\eta-\theta)} \mathrm{d} \sigma\right)^{p / 2} .
\end{aligned}
$$

We apply Young's inequality to get

$$
\begin{aligned}
\left\|y_{\eta}\right\|_{L_{\mathcal{F}}^{p}\left(\Omega ; L^{p}(0, T ; X)\right)}^{p}= & \left(\mathbb{E} \int_{0}^{T}\left|y_{\eta}(\tau)\right|^{p} \mathrm{~d} \tau\right) \\
\leq & C \mathbb{E}\left[\left(\int_{0}^{T} e^{-2 \beta \sigma}\|g(\sigma, J(v(\sigma)))\|_{L_{2}(U, H)}^{2} \mathrm{~d} \sigma\right)^{2 / p}\right]^{p / 2} \\
& \left(\int_{0}^{\infty} e^{-\tau}(2 \beta)^{1+2 \gamma-2(\theta-\eta)} \tau^{-2(\gamma+1+\eta-\theta)} \mathrm{d} \tau\right)^{p / 2},
\end{aligned}
$$

Copyright $@$ by SIAM. Unauthorized reproduction of this article is prohibited. 
where the last integral is obtained by a change of variables. Hence, for any $\gamma<$ $(\theta-\eta)-1 / 2$ (notice that we can always choose $\gamma>0$ small enough such that this holds) we obtain

$$
\begin{aligned}
& \left\|y_{\eta}\right\|_{L_{\mathcal{F}}^{p}\left(\Omega ; L^{p}(0, T ; H)\right)} \\
& \quad \leq C_{T}(2 \beta)^{\frac{1}{2}+\gamma-(\theta-\eta)}\left(\mathbb{E}\left[\int_{0}^{T} e^{-p \beta \sigma}\|g(\sigma, J(v(\sigma)))\|_{L_{2}(U, H)}^{p} \mathrm{~d} \sigma\right]\right)^{1 / p} .
\end{aligned}
$$

Now, taking into account the assumptions on $g$ and the choice of $v \in L_{\mathcal{F}}^{p}(\Omega ; C([0, T]$; $\left.X_{\eta}\right)$ ), we estimate the integral term above and we finally arrive at

$$
\left\|y_{\eta}\right\|_{L_{\mathcal{F}}^{p}\left(\Omega ; L^{p}(0, T ; X)\right)} \leq C(2 \beta)^{\frac{1}{2}+\gamma-(\theta-\eta)} .
$$

It follows that in particular $y_{\eta} \in L^{p}(0, T ; X), \mathbb{P}$-a.s.

Step 2. In [11, Appendix A] it is proved that for any $\gamma \in(0,1), p$ large enough such that $\gamma-\frac{1}{p}>0$, the linear operator

$$
R_{\gamma} \phi(t)=\int_{0}^{t}(t-\sigma)^{\gamma-1} e^{(t-\sigma) B} \phi(\sigma) \mathrm{d} \sigma
$$

is a bounded operator from $L^{p}(0, T ; X)$ into $C([0, T] ; X)$. Using this result in Step 1, the thesis follows.

Theorem 3.3. Under Assumptions 2.2 and 3.1, for every $p \geq 2$ there exists a unique process $v \in L_{\mathcal{F}}^{p}\left(\Omega ; C\left([0, T] ; X_{\eta}\right)\right)$ solution of $(3.2)$. Moreover, the solution $v\left(t ; s, v_{0}\right)$ depends continuously on the initial conditions $\left(s, v_{0}\right) \in[0, T] \times X_{\eta}$, and the estimate

$$
\mathbb{E} \sup _{t \in[s, T]}\|v(t)\|_{\eta}^{p} \leq C\left(1+\left\|v_{0}\right\|_{\eta}\right)^{p}
$$

holds for some constant $C$ depending on $T$ and the parameters of the problem.

Proof. The first part of the proof proceeds, basically, on the same lines as the proof of Theorem 3.2 in [5]. We have to prove that the mapping $\mathcal{K}$ from $L_{\mathcal{F}}^{p}\left(\Omega ; C\left([0, T] ; X_{\eta}\right)\right)$ to itself given by

$$
\mathcal{K}(v)(t)=e^{(t-s) B} v_{0}+\int_{s}^{t} e^{(t-\tau) B}(I-B) P g(\tau, J(v(\tau))) \mathrm{d} W(\tau), \quad t \in[s, T],
$$

is a contraction under the following norm:

$$
\|v\|_{\eta}^{p}=\mathbb{E} \sup _{t \in[0, T]} e^{-\beta p t}\|v(t)\|_{\eta}^{p} .
$$

Lemma 3.2 shows that for every $\frac{1}{p}<\theta-\eta-\frac{1}{2}$ the operator $\Lambda$ is a well-defined mapping in the space $L_{\mathcal{F}}^{p}\left(\Omega ; C\left([0, T] ; X_{\eta}\right)\right)$. In order to conclude the proof that $\mathcal{K}$ maps $L_{\mathcal{F}}^{p}\left(\Omega ; C\left([0, T] ; X_{\eta}\right)\right)$ into itself it is sufficient to recall that the initial condition $v_{0}$ belongs to $X_{\eta}$, hence $t \mapsto e^{(t-s) B} v_{0}$, extended to a constant for $t<s$,

$$
S(t-s) v_{0}=v_{0} \quad \text { for } t<s
$$

belongs to $L_{\mathcal{F}}^{p}\left(\Omega ; C\left([0, T] ; X_{\eta}\right)\right)$.

Copyright $@$ by SIAM. Unauthorized reproduction of this article is prohibited. 
Next, we prove that $\mathcal{K}$ is a contraction in the space $L_{\mathcal{F}}^{p}\left(\Omega ; C\left([0, T] ; X_{\eta}\right)\right)$. If $v, v_{1}$ are processes belonging to this space, passages similar to those in Lemma 3.2 show that

$$
\left\|\Lambda(v)-\Lambda\left(v_{1}\right)\right\|_{\eta} \leq C(2 \beta)^{\frac{1}{2}+\gamma-(\theta-\eta)}\left\|v-v_{1}\right\|_{\eta} .
$$

Moreover, we can find $\beta$ large enough such that

$$
C(2 \beta)^{\frac{1}{2}+\gamma-(\theta-\eta)} \leq \delta<1
$$

so that $\mathcal{K}$ becomes a contraction on the time interval $[0, T]$ and by a classical fixed point argument we get that there exists a unique solution of the mild equation (3.3) on $[0, T]$.

Since the solution to (3.3) verifies $v=\mathcal{K}(v)$ we also deduce from the above computations that

$$
\|v\|_{\eta}=\|\mathcal{K}(v)\|_{\eta} \leq \delta+C(T)\left\|v_{0}\right\|_{\eta}
$$

hence

$$
\|v\|_{\eta} \leq C\left(1+\left\|v_{0}\right\|_{\eta}\right)
$$

In the last part of the proof we are concerned with the regular dependence of the solution on the initial conditions. The results in this section rely on Proposition 2.4 in [21], where a parameter depending contraction principle is provided.

As before, we introduce the map

$$
\mathcal{K}(v, s, x)(t)=e^{(t-s) B} v_{0}+\Lambda(v, s)(t)
$$

and we set $\mathcal{K}\left(v, s, v_{0}\right)(t)=v_{0}$ for $t<s$. For a suitable $T>0$, we have shown that this mapping is a contraction in the space $L_{\mathcal{F}}^{p}\left(\Omega ; C\left([0, T] ; X_{\eta}\right)\right)$. Now the thesis follows if we prove that for every $v \in L_{\mathcal{F}}^{p}\left(\Omega ; C\left([0, T] ; X_{\eta}\right)\right)$, the mapping $\left(s, v_{0}\right) \mapsto \mathcal{K}\left(v, s, v_{0}\right)$ is continuous as a map from $[0, T] \times X_{\eta} \rightarrow L_{\mathcal{F}}^{p}\left(\Omega ; C\left([0, T] ; X_{\eta}\right)\right)$.

We introduce two sequences $\left\{s_{n}^{-}\right\}$and $\left\{s_{n}^{+}\right\}$such that $s_{n}^{-} \nearrow s$ and $s_{n}^{+} \searrow s$. First, recalling that we extend $S(t-s) v_{0}=v_{0}$ for $t<s$, we have

$$
\sup _{t \in[0, T]}\left\|e^{\left(t-s_{n}^{+}\right) B} v_{0}-e^{\left(t-s_{n}^{-}\right) B} v_{0}\right\|_{\eta}=\sup _{t \in\left[s_{n}^{-}, T\right]}\left\|e^{\left(t-s_{n}^{+}\right) B}\left[v_{0}-e^{\left(s_{n}^{+}-s_{n}^{-}\right) B} v_{0}\right]\right\|_{\eta} \rightarrow 0
$$

and also the map $v_{0} \rightarrow\left\{t \mapsto e^{(t-s) B} v_{0}\right\}$ is clearly continuous from $X_{\eta}$ into $C\left([0, T] ; X_{\eta}\right)$.

Next, we consider the mapping $\Lambda$. Recall that

$$
\Lambda(v, s)(t)=\int_{s}^{t} e^{(t-\tau) B}(I-B) P g(\tau, J(v(\tau))) \mathrm{d} W(\tau)
$$

and we set $\Lambda(v, s)(t)=0$ for $t<s$. Our aim is to prove that

$$
\mathbb{E} \sup _{t \in[0, T]}\left\|\Lambda\left(v, s_{n}^{+}\right)(t)-\Lambda\left(v, s_{n}^{-}\right)(t)\right\|_{\eta}^{p} \longrightarrow 0 .
$$

If $t<s_{n}^{-}$, then both terms are zero. For $s_{n}^{-}<t<s_{n}^{+}$, the first term disappears and only the second one remains. Finally, for $t>s_{n}^{+}$the first integral compensates a part 
of the second one and we get

$$
\begin{aligned}
\mathbb{E} \sup _{t \in[0, T]} & \left\|\Lambda\left(v, s_{n}^{+}\right)(t)-\Lambda\left(v, s_{n}^{-}\right)(t)\right\|_{\eta}^{p} \\
= & \mathbb{E} \sup _{t \in[0, T]}\left\|\int_{s_{n}^{-}}^{s_{n}^{+}} e^{(t-\tau) B}(I-B) \operatorname{Pg}(\tau, J(v(\tau))) \mathrm{d} W(\tau)\right\|_{\eta}^{p} \\
= & \mathbb{E} \sup _{t \in[0, T]}\left\|\int_{0}^{t} 1_{s_{n}^{-} \leq \tau \leq s_{n}^{+}} e^{(t-\tau) B}(I-B) \operatorname{Pg}(\tau, J(v(\tau))) \mathrm{d} W(\tau)\right\|_{\eta}^{p} .
\end{aligned}
$$

Now using factorization method we can write

$$
\begin{aligned}
(I-B)^{\eta} \int_{0}^{t} 1_{s_{n}^{-} \leq \tau \leq s_{n}^{+}} e^{(t-\tau) B} & (I-B) P g(\tau, J(v(\tau))) \mathrm{d} W(\tau) \\
& =c_{\gamma} \int_{0}^{t} 1_{s_{n}^{-} \leq \tau \leq s_{n}^{+}} e^{-\beta(t-\tau)}(t-\tau)^{\gamma-1} e^{(t-\tau) B} y_{\eta}(\tau) \mathrm{d} \tau,
\end{aligned}
$$

where $y_{\eta}$ is given by (3.4).

Arguing as in proof of Lemma 3.2 we see that $1_{s_{n}^{-} \leq \tau \leq s_{n}^{+}} y_{\eta}(\tau) \in L^{p}((0, T) ; X)$. Since (see [11, Appendix A]) for any $\gamma \in(0,1), p$ large enough such that $\gamma-\frac{1}{p}>0$, the linear operator $R_{\gamma}$ defined by (3.7) is a bounded operator from $L^{p}(0, T ; X)$ into $C([0, T] ; X)$, we have

$$
\begin{aligned}
& \mathbb{E} \sup _{t \in[0, T]}\left\|\Lambda\left(v, s_{n}^{+}\right)-\Lambda\left(v, s_{n}^{-}\right)\right\|_{\eta}^{p} \\
& \leq c \mathbb{E} \sup _{t \in[0, T]}\left|\int_{0}^{t}(t-\tau)^{\gamma-1} e^{(t-\tau) B_{1}} 1_{s_{n}^{-} \leq \tau \leq s_{n}^{+}} y_{\eta}(\tau) \mathrm{d} \tau\right|^{p} \\
& \leq c\left\|R_{\gamma}\right\|^{p} \mathbb{E} \int_{0}^{T}\left|1_{s_{n}^{-} \leq \tau \leq s_{n}^{+}} y_{\eta}(\tau)\right|^{p} \mathrm{~d} \tau \rightarrow 0,
\end{aligned}
$$

where the final convergence comes as an immediate consequence of the dominated convergence theorem. Collecting all the above estimates, the continuity of the mapping $\left(s, v_{0}\right) \mapsto \mathcal{K}\left(v, s, v_{0}\right)$ is proved.

3.1. The general case. The last result in this section provides the existence, for every admissible control, of the solution for the stochastic problem (1.2), where we identify the nonlinear mappings $F$ and $G$, within the state space approach, with $g(t, J(v(t))) r(t, J(v(t)), \gamma(t))$ and $g(t, J(v(t)))$, respectively:

$$
\begin{aligned}
\mathrm{d} v(t)= & {[B v(t)+(I-B) P g(t, J(v(t))) r(t, J(v(t)), \gamma(t))] \mathrm{d} t } \\
& +(I-B) P g(t, J(v(t))) \mathrm{d} W(t), \\
v(s)= & v_{0} .
\end{aligned}
$$

At this stage, it is necessary to make precise the assumptions on the control process $\gamma$ and the control operator $r$ that enters in this problem.

Assumption 3.4.

(i) An admissible control $\gamma$ is a stochastic process $\gamma: \Omega \times[0, T] \rightarrow \Xi$ that is $\mathcal{F}_{t}$-adapted and square integrable,

$$
\mathbb{E} \int_{0}^{T}|\gamma(s)|^{2} \mathrm{~d} s<+\infty .
$$

Copyright $@$ by SIAM. Unauthorized reproduction of this article is prohibited. 
This square summability requirement is intuitively justified by the idea that a control process which is not square summable would have infinite cost.

(ii) The function $r:[0, T] \times H \times \Xi \rightarrow U$ is measurable and there exists $C>0$ such that for $t \in[0, T], u, u^{\prime} \in H$, and $\gamma \in \Xi$, it holds that

$$
\left|r(t, u, \gamma)-r\left(t, u^{\prime}, \gamma\right)\right| \leq C(1+|\gamma|)\left|u-u^{\prime}\right|
$$

moreover, $r(t, u, \cdot)$ has sublinear growth uniformly in $(t, u) \in[0, T] \times H$ : there exists $C>0$ such that for $t \in[0, T]$ and $u \in H$, it holds that

$$
|r(t, u, \gamma)| \leq C(1+|\gamma|) .
$$

Finally, for all $t \in[0, T], u \in H, r(t, u, \cdot)$ is a continuous function from $\Xi$ to $U$.

TheOREM 3.5. Under Assumptions 2.2, 3.1, and 3.4, let $\gamma$ be an admissible control. Then there exists a unique mild solution $v$ of $(3.9)$ with $v \in L_{\mathcal{F}}^{2}\left(\Omega ; C\left([0, T] ; X_{\eta}\right)\right)$.

Proof. We shall use an approximation procedure in order to handle the growth bound of the function $r(t, J(v), \gamma)$ in $\gamma$. We introduce the sequence of stopping times

$$
\tau_{n}=\inf \left\{\left.t \in[0, T]\left|\int_{0}^{t}\right| \gamma(s)\right|^{2} \mathrm{~d} s>n\right\}
$$

with the convention that $\tau_{n}=T$ if the indicated set is empty. Since $\gamma$ is an admissible control, i.e., $\mathbb{E} \int_{0}^{T}|\gamma(s)|^{2} \mathrm{~d} s<+\infty$, for $\mathbb{P}$-almost every $\omega \in \Omega$ there exists an integer $N(\omega)$ such that

$$
n \geq N(\omega) \text { implies } \tau_{n}(\omega)=T .
$$

Let us fix $\gamma_{0} \in \Xi$ and let us define, for every $n \in \mathbb{N}$,

$$
\gamma_{n}(t)=\gamma(t) 1_{t \leq \tau_{n}}+\gamma_{0} 1_{t \geq \tau_{n}}
$$

and we consider the family of equations

$$
\begin{aligned}
\mathrm{d} v_{n}(t)= & {\left[B v_{n}(t)+(I-B) P g\left(t, J\left(v_{n}(t)\right)\right) r\left(t, J\left(v_{n}(t)\right), \gamma_{n}(t)\right)\right] \mathrm{d} t } \\
& +(I-B) \operatorname{Pg}\left(t, J\left(v_{n}(t)\right)\right) \mathrm{d} W_{t}, \\
v_{n}(s)= & v_{0} .
\end{aligned}
$$

For simplicity of notation we fix the initial time $s=0$; the solution $v_{n}$ of (3.12) is the fixed point of the mapping

$$
v \mapsto \mathcal{K}_{n}\left(v, v_{0}\right)=e^{t B} v_{0}+\Lambda(v)(t)+\Gamma_{n}(v)(t),
$$

where $\Gamma_{n}(v)$ is the process defined by

$$
\Gamma_{n}(v)(t)=\int_{0}^{t} e^{(t-\tau) B}(I-B) \operatorname{Pg}(\tau, J(v(\tau))) r\left(\tau, J(v(\tau)), \gamma_{n}(\tau)\right) \mathrm{d} \tau .
$$

In Lemma 3.7 we prove that for every $p$ the operator $\Gamma_{n}$ is a well-defined mapping on $L_{\mathcal{F}}^{p}\left(\Omega ; C\left([0, T], e^{-\beta t} ; X_{\eta}\right)\right)$ and that it is a contraction for every $\beta$ large enough. Putting together the estimates (3.8) and (3.14) concerning the mappings $\Lambda$ and $\Gamma_{n}$ we obtain that the mapping $v \mapsto \mathcal{K}_{n}(v, x)$ is a contraction on the space 
$L_{\mathcal{F}}^{p}\left(\Omega ; C\left([0, T] ; X_{\eta}\right)\right)$ and for every $n \in \mathbb{N}$ there exists a unique solution $v_{n}$ of the approximate problem (3.12).

Notice that $v_{n}(t)=v_{n+1}(t)$ coincide on the time interval $\left[0, \tau_{n}\right]$ and $\tau_{n} \nearrow T$ almost surely as $n \rightarrow \infty$. Hence we can define a process $v(t)$ as

$$
v(t)=v_{n}(t) \text { on }\left[0, \tau_{n}\right]
$$

and clearly $v$ is the required solution of (3.9). that

It remains to prove that $v \in L_{\mathcal{F}}^{2}\left(\Omega ; C\left([0, T] ; X_{\eta}\right)\right.$. The claim follows if we prove

$$
\sup _{n \in \mathbb{N}} \mathbb{E} \sup _{t \leq T}\left\|v_{n}(t)\right\|_{\eta}^{2}<\infty
$$

From the mild form of (3.12) we obtain

$$
\begin{aligned}
v_{n}(t)= & e^{t B} x+\int_{0}^{t} e^{(t-\sigma) B}(I-B) P g\left(\sigma, J v_{n}(\sigma)\right) r\left(\sigma, J v_{n}(\sigma), \gamma_{n}(\sigma)\right) \mathrm{d} \sigma \\
& +\int_{0}^{t} e^{(t-\sigma) B}(I-B) \operatorname{Pg}\left(\sigma, J v_{n}(\sigma)\right) \mathrm{d} W(\sigma) .
\end{aligned}
$$

We first notice that the first integral term on the right-hand side can only be estimated in $L^{2}(\Omega)$-norm,

$$
\begin{gathered}
\mathbb{E} \sup _{t \leq T}\left\|\int_{0}^{t} e^{(t-\sigma) B}(I-B) P g\left(\sigma, J v_{n}(\sigma)\right) r\left(\sigma, J v_{n}(\sigma), \gamma_{n}(\sigma)\right) \mathrm{d} \sigma\right\|_{\eta}^{2} \\
\leq \mathbb{E} \sup _{t \leq T}\left|\int_{0}^{t}(t-\sigma)^{-(1+\eta-\theta)}\left(1+\left|\gamma_{n}(\sigma)\right|\right) \mathrm{d} \sigma\right|^{2} \\
\leq C T^{2(\theta-\eta)-1}\left(\int_{0}^{T}\left(1+\mathbb{E}|\gamma(s)|^{2}\right) \mathrm{d} s\right) \leq C,
\end{gathered}
$$

thanks to the assumptions on the admissible control $\gamma$.

As far as the stochastic term is concerned, we can give the estimate also in the $L^{2 p}(\Omega)$-norm for $p \geq 1$; although we only need $p=1$ here, the general case will be useful later. Using the factorization method as in proof of Lemma 3.2 we have that

$$
\begin{aligned}
\mathbb{E} \sup _{t \leq T}\left\|\int_{0}^{t} e^{(t-\sigma) B}(I-B) P g\left(\sigma, J v_{n}(\sigma)\right) \mathrm{d} W(\sigma)\right\|_{\eta}^{2 p} \\
=\mathbb{E} \sup _{t \leq T}\left\|c_{\gamma} \int_{0}^{t} e^{-\beta(t-\tau)}(t-\tau)^{\gamma-1} e^{(t-\tau) B} y_{\eta}^{n}(\tau) \mathrm{d} \tau\right\|^{2 p},
\end{aligned}
$$

where $y_{\eta}^{n}$ is the process

$$
y_{\eta}^{n}(\tau)=\int_{0}^{\tau} e^{-\beta \sigma}(\tau-\sigma)^{-\gamma} e^{-\beta(\tau-\sigma)} e^{(\tau-\sigma) B}\left(I-B_{-1}\right)^{\eta} P g\left(\sigma, J\left(v_{n}(\sigma)\right)\right) \mathrm{d} W(\sigma) .
$$

With the same calculation as in proof of Lemma 3.2 (see (3.5), (3.6)), we get

$$
\left\|y_{\eta}^{n}\right\|_{L_{\mathcal{F}}^{p}\left(\Omega ; L^{p}(0, T ; X)\right)} \leq C
$$

Copyright $@$ by SIAM. Unauthorized reproduction of this article is prohibited. 
where the constant $C$ is independent on $n$. Hence, applying Hölder's inequality in the (3.13), we obtain

$$
\mathbb{E} \sup _{t \leq T}\left\|\int_{0}^{t} e^{(t-\sigma) B}(I-B) P g\left(\sigma, J v_{n}(\sigma)\right) \mathrm{d} W(\sigma)\right\|_{\eta}^{2 p} \leq C \mathbb{E} \int_{0}^{T}\left|y_{\eta}^{n}(\sigma)\right|^{2 p} \mathrm{~d} \sigma \leq C,
$$

which is bounded, independently of $n$, using the bound of $g$ in Assumption 3.1. We have thus proved the thesis.

COROLlary 3.6. The family of random variables $\sup _{t \leq T}\left\|v_{n}(t)\right\|_{\eta}^{2}$ is uniformly integrable.

Proof. Proceeding as above, we have that

$$
\sup _{t \leq T}\left\|v_{n}(t)\right\|_{\eta}^{2} \leq C\left(1+\int_{0}^{T}|\gamma(s)|^{2} \mathrm{~d} s+\sup _{t \leq T}\left\|\Lambda\left(v_{n}, t\right)\right\|_{\eta}^{2}\right)
$$

and we notice that the first two terms are integrable; the last is a uniformly integrable family of random variables, since we have proved in (3.13) that it is uniformly bounded in $L^{2 p}(\Omega)$-norm for some $p>1$. Therefore, also $\sup _{t \leq T}\left\|v_{n}(t)\right\|_{\eta}^{2}$ is uniformly integrable and the claim is proved.

Lemma 3.7. For every $p$ the operator $\Gamma_{n}$ maps $L_{\mathcal{F}}^{p}\left(\Omega ; C\left([0, T], e^{-\beta t} ; X_{\eta}\right)\right)$ into itself and it is a contraction for every $\beta$ large enough.

Proof. As in the proof of Lemma 3.2, we consider only the case $s=0$ and we write $\Gamma_{n}(t)$ for $\Gamma_{n}(v)(t)$ for $v \in L_{\mathcal{F}}^{p}\left(\Omega ; C\left([0, T] ; X_{\eta}\right)\right)$. The following computation leads to the thesis:

$$
\begin{aligned}
\mathbb{E} \sup _{t \in[0, T]} e^{-\beta p t}\left\|\Gamma_{n}(v(t))-\Gamma_{n}\left(v^{\prime}(t)\right)\right\|_{\eta}^{p} \\
\leq \mathbb{E} \mid \sup _{t \in[0, T]} \int_{0}^{t} e^{-\beta \sigma} \| e^{-\beta(t-\sigma)} e^{(t-\sigma) B}(I-B)^{1+\eta} \\
\quad \cdot P\left[g(\sigma, J(v(\sigma))) r\left(\sigma, J(v(\sigma)), \gamma_{n}\right)-g\left(\sigma, J\left(v^{\prime}(\sigma)\right)\right) r\left(\sigma, J\left(v^{\prime}(\sigma)\right), \gamma_{n}\right)\right] \|\left.\mathrm{d} \sigma\right|^{p} \\
\leq\left(\int_{0}^{\infty} e^{-2 \beta \sigma} \sigma^{-2(1+\eta-\theta)} \mathrm{d} \sigma\right)^{p / 2} \\
\cdot \mathbb{E}\left(\int_{0}^{T} \| e^{-\beta \sigma}\left[g(\sigma, J(v(\sigma))) r\left(\sigma, J(v(\sigma)), \gamma_{n}(\sigma)\right)\right.\right. \\
\leq C\left(\Gamma[2(\theta-\eta)-1](2 \beta)^{1-2(\theta-\eta)}\right)^{p / 2} \\
\quad \mathbb{E}\left(\int_{0}^{T} e^{-\beta \sigma}\left(|1+| \gamma_{n}(\sigma) \mid\right)^{2}\left\|v(\sigma)-v^{\prime}(\sigma)\right\|_{\eta}^{2} \mathrm{~d} \sigma\right)^{p / 2} \\
\leq C \mathbb{E} \sup _{t \in[0, T]} e^{-\beta p t}\left\|v(\sigma)-v^{\prime}(\sigma)\right\|_{\eta}^{p}\left(\int_{0}^{T} e^{\beta \sigma}\left(|1+| \gamma_{n}(\sigma) \mid\right)^{2} \mathrm{~d} \sigma\right)^{p / 2}
\end{aligned}
$$

Copyright $@$ by SIAM. Unauthorized reproduction of this article is prohibited. 
by the construction of $\tau_{n}$ it holds that

$$
\int_{0}^{T}\left(1+\left|\gamma_{n}(\sigma)\right|\right)^{2} \mathrm{~d} \sigma \leq C\left(1+n+\left|\gamma_{0}\right|^{2}\right)=: \tilde{C} \quad \mathbb{P}-\text { a.s. }
$$

and it follows that

$$
\leq(2 \beta)^{\frac{p}{2}(1-2(\theta-\eta))} e^{\frac{p}{2} \beta T} \tilde{C}^{\frac{p}{2}}\left\|v-v^{\prime}\right\|_{\eta}^{p} .
$$

With the same computation we obtain that $\Gamma_{n}$ is well defined:

$$
\begin{aligned}
& \left\|\Gamma_{n}(v)\right\|_{\eta}^{p} \leq C\left(\Gamma[2(\theta-\eta)-1](2 \beta)^{1-2(\theta-\eta)}\right)^{p / 2} \mathbb{E}\left(\int_{0}^{T} e^{-\beta \sigma}\left(|1+| \gamma_{n}(\sigma) \mid\right)^{2} \mathrm{~d} \sigma\right)^{p / 2} \\
& (3.14) \quad \leq K(2 \beta)^{\frac{p}{2}(1-2(\theta-\eta))} .
\end{aligned}
$$

Hence $\Gamma_{n}$ is a contraction with norm decreasing with $\beta$.

4. The solution of the controlled stochastic Volterra equation. Following the preparatory results proved in the previous section, we prove that the main Theorem 4.2 of existence and uniqueness of solutions of the original controlled Volterra equation (1.1). Obviously, as a special case of the main theorem we also get that (3.1) has a unique solution.

The proof of this result relies again on the approximation procedure introduced in section 3. As in the proof of Theorem 3.5, we introduce the sequence of stopping times

$$
\tau_{n}=\inf \left\{\left.t \in[0, T]\left|\int_{0}^{t}\right| \gamma(s)\right|^{2} \mathrm{~d} s>n\right\}
$$

with the convention that $\tau_{n}=T$ if the indicated set is empty. Since $\gamma$ is an admissible control, i.e., $\mathbb{E} \int_{0}^{T}|\gamma(s)|^{2} \mathrm{~d} s<+\infty$, for $\mathbb{P}$-almost every $\omega \in \Omega$ there exists an integer $N(\omega)$ such that

$$
n \geq N(\omega) \text { implies } \tau_{n}(\omega)=T .
$$

Let us fix $\gamma_{0} \in \Xi$ and let us define for every $n \in \mathbb{N}$

$$
\gamma_{n}(t)=\gamma(t) 1_{t \leq \tau_{n}}+\gamma_{0} 1_{t \geq \tau_{n}}
$$

and consider the family of equations

$$
\begin{aligned}
\mathrm{d} v_{n}(t)= & {\left[B v_{n}(t)+(I-B) P g\left(t, J\left(v_{n}(t)\right)\right) r\left(t, J\left(v_{n}(t)\right), \gamma_{n}(t)\right)\right] \mathrm{d} t } \\
& +(I-B) P g\left(t, J\left(v_{n}(t)\right)\right) \mathrm{d} W_{t}, \\
v_{n}(s)= & v_{0} .
\end{aligned}
$$

We focus our attention on the following result, which will give us the main tool to prove Theorem 4.2 .

TheOREM 4.1. Under Assumptions 2.2, 3.1, and 3.4, let $\gamma$ be an admissible control. For every $n \in \mathbb{N}$, the process

$$
u_{n}(t)= \begin{cases}u_{0}(t), & t \leq 0, \\ J v_{n}(t), & t \in[0, T],\end{cases}
$$

Copyright $@$ ㅇ by SIAM. Unauthorized reproduction of this article is prohibited. 
where $v_{n}$ is the solution of problem (4.2), is the unique solution of the equation

$$
\begin{aligned}
\frac{d}{d t} \int_{-\infty}^{t} a(t-s) u(s) \mathrm{d} s & =A u(t)+g(t, u(t))\left[r\left(t, u(t), \gamma_{n}(t)\right)+\dot{W}(t)\right], \\
u(t) & =u_{0}(t), \quad t \leq 0,
\end{aligned}
$$

where $\gamma_{n}$ is the control process defined in (4.1).

Proof. We propose to fulfill the following steps.

Step I. The affine equation

$$
\begin{aligned}
\frac{d}{d t} \int_{-\infty}^{t} a(t-s) u(s) \mathrm{d} s & =A u(t)+g(t, \tilde{u}(t))\left[r\left(t, \gamma_{n}(t), \tilde{u}(t)\right)+\dot{W}(t)\right], \\
u(t) & =u_{0}(t), \quad t \leq 0,
\end{aligned}
$$

defines a contraction mapping $\mathcal{Q}: \tilde{u} \mapsto u$ on the space $L_{\mathcal{F}}^{2}(\Omega ; C([0, T] ; H))$. Therefore, (4.4) admits a unique solution.

Step II. The process $u_{n}$ defined in (4.3) satisfies (4.4). Accordingly, by the uniqueness of the solution, the thesis of the theorem follows.

Proof of Step I. We proceed to define the mapping

$$
\mathcal{Q}: L^{p}(\Omega ; C([0, T] ; H)) \rightarrow L^{p}(\Omega ; C([0, T] ; H)),
$$

where $\mathcal{Q}(\tilde{u})=u$ is the solution of the problem

$$
\begin{aligned}
\frac{d}{d t} \int_{-\infty}^{t} a(t-s) u(s) \mathrm{d} s & =A u(t)+g(t, \tilde{u}(t))\left[r\left(t, \tilde{u}(t), \gamma_{n}(t)\right)+\dot{W}(t)\right], \\
u(t) & =u_{0}(t), \quad t \leq 0 .
\end{aligned}
$$

Let $\tilde{u}_{1}, \tilde{u}_{2}$ be two processes belonging to $L^{p}(\Omega ; C([0, T] ; H))$ and take $u_{1}=\mathcal{Q}\left(\tilde{u}_{1}\right)$ and $u_{2}=\mathcal{Q}\left(\tilde{u}_{2}\right)$. It follows from the uniqueness of the solution, proved in Proposition 2.7, that the solution $u_{i}(t)(i=1,2)$ has the representation

$$
u_{i}(t)= \begin{cases}J v_{i}(t), & t \in[0, T] \\ u_{0}(t), & t \leq 0\end{cases}
$$

where

$$
\begin{aligned}
v_{i}(t)= & e^{t B} v_{0}+\int_{0}^{t} e^{(t-s) B}(I-B) P g\left(s, \tilde{u}_{i}(s)\right) r\left(s, \tilde{u}_{i}(s), \gamma_{n}(s)\right) \mathrm{d} s \\
& +\int_{0}^{t} e^{(t-s) B}(I-B) \operatorname{Pg}\left(s, \tilde{u}_{i}(s)\right) \mathrm{d} W(s) .
\end{aligned}
$$

In particular,

$$
U(t)=u_{1}(t)-u_{2}(t)= \begin{cases}J\left(v_{1}(t)-v_{2}(t)\right), & t \in[0, T] \\ 0, & t \leq 0\end{cases}
$$

then

$$
\mathbb{E} \sup _{t \in[0, T]} e^{-\beta p t}|U(t)|^{p} \leq\|J\|_{L\left(X_{\eta}, H\right)}^{p} \mathbb{E} \sup _{t \in[0, T]} e^{-\beta p t}\left\|v_{1}(t)-v_{2}(t)\right\|_{\eta}^{p}
$$

Copyright $@$ ( ) by SIAM. Unauthorized reproduction of this article is prohibited. 
and the quantity on the right-hand side can be treated as in Theorem 3.5 and the claim follows.

Proof of Step II. It follows from the previous step that there exists at most a unique solution $u_{n}$ of problem (4.4); in order to prove Theorem 4.1 it only remains to prove the representation formula (4.3).

Let $\tilde{f}(s)=g\left(s, J v_{n}(s)\right) r\left(s, J v_{n}(s), \gamma_{n}(s)\right)$ and $\tilde{g}(s)=g\left(s, J v_{n}(s)\right)$; it is a consequence of Theorem 2.7 that $u$, defined in (4.3), is a weak solution of the problem

$$
\begin{aligned}
\frac{d}{d t} \int_{-\infty}^{t} a(t-s) u(s) \mathrm{d} s & =A u(t)+\tilde{f}(t)+\tilde{g}(t) \dot{W}(t), \quad t \in[0, T], \\
u(t) & =u_{0}(t), \quad t \leq 0,
\end{aligned}
$$

and the definition of $\tilde{f}$ and $\tilde{g}$ implies that $u$ is a weak solution of

$$
\begin{aligned}
\frac{d}{d t} \int_{-\infty}^{t} a(t-s) u(s) \mathrm{d} s & =A u(t)+g\left(s, J v_{n}(s)\right)\left[r\left(s, J v_{n}(s), \gamma_{n}(s)\right)+\dot{W}(t)\right], \\
u(t) & =u_{0}(t), \quad t \leq 0,
\end{aligned}
$$

that is problem (4.4).

Now we are in position to prove the main theorem of this section.

TheOREM 4.2. Under Assumptions 2.2, 3.1, and 3.4, let $v$ be the solution to problem (3.9) whose existence is proved in Theorem 3.5. Then for every admissible control $\gamma$, the process

$$
u(t)= \begin{cases}u_{0}(t), & t \leq 0, \\ J v(t), & t \in[0, T],\end{cases}
$$

is the unique solution of the stochastic Volterra equation

$$
\begin{aligned}
\frac{d}{d t} \int_{-\infty}^{t} a(t-s) u(s) \mathrm{d} s & =A u(t)+g(t, u(t))[r(t, u(t), \gamma(t))+\dot{W}(t)], \quad t \in[0, T], \\
u(t) & =u_{0}(t), \quad t \leq 0 .
\end{aligned}
$$

Proof. Theorem 4.1 states the existence of a family of processes $\left\{u_{n}(t), t \in\right.$ $[0, T]\}_{n \in \mathbb{N}}$, solutions to the stochastic Volterra equations (4.4), and such that $u_{n}(t)=$ $u_{n+1}(t)$ on the time interval $\left[0, \tau_{n}\right]$ and $\tau_{n} \nearrow T$ almost surely as $n \rightarrow \infty$. Hence we can define a process $u(t)$ as

$$
u(t)=u_{n}(t) \text { on }\left[0, \tau_{n}\right]
$$

and clearly $u$ is the required solution of (4.5). Further, by the uniqueness of the solution, it follows that $u(t)=J v(t)$ for $t \in[0, T]$, where $v$ is the process constructed in Theorem 3.5.

It remains to verify that $u \in L_{\mathcal{F}}^{2}(\Omega ; C([0, T] ; H))$. This follows from the representation $u(t)=J v(t)$ for $t \in[0, T]$ and from the fact-proved in Theorem 3.5-that $v \in L_{\mathcal{F}}^{2}\left(\Omega ; C\left([0, T] ; X_{\eta}\right)\right)$.

5. The optimal control problem in the state space setting. We proceed with a study of the optimal control problem associated to the stochastic Volterra 
equation (1.1). We showed in section 3 that we can associate to such equation

$$
\begin{aligned}
\frac{d}{d t} \int_{-\infty}^{t} a(t-s) u(s) \mathrm{d} s & =A u(t)+g(t, u(t))[r(t, u(t), \gamma(t))+\dot{W}(t)], \\
u(t) & =u_{0}(t), \quad t \leq 0,
\end{aligned}
$$

the controlled state equation

$$
\begin{aligned}
\mathrm{d} v(t)= & {[B v(t)+(I-B) P g(t, J(v(t))) r(t, J(v(t)), \gamma(t))] \mathrm{d} t } \\
& +(I-B) P g(t, J(v(t))) \mathrm{d} W_{t}, \\
v(0)= & v_{0} .
\end{aligned}
$$

Hence the original control problem which consists of minimizing a cost functional of the form

$$
\mathbb{E} \int_{0}^{T} l(t, u(t), \gamma(t)) \mathrm{d} t+\mathbb{E}[\phi(u(T))]
$$

can be translated in the state space setting: we consider the optimal control problem given by a state equation of the form (3.9) and a cost functional of the form

$$
\mathbb{J}\left(v_{0}, \gamma\right)=\mathbb{E} \int_{0}^{T} l(t, J(v(t)), \gamma(t)) \mathrm{d} t+\mathbb{E}[\phi(J(v(T)))] .
$$

Assumption 5.1.

(i) The functions $l$ and $\phi$, which enter the definition of the cost functional, are measurable mappings $l:[0, T] \times H \times \Xi \rightarrow R, \phi: H \rightarrow \mathbb{R}$, satisfying the bounds

$$
0 \leq l(t, u, \gamma) \leq C\left(1+|u|^{2}+|\gamma|^{2}\right)
$$

and

$$
0 \leq \phi(u) \leq C\left(1+|u|^{2}\right)
$$

for given constants $C$.

Moreover, for all $t \in[0, T], u \in H, l(t, u, \cdot)$ is a continuous function from $\Xi$ to $\mathbb{R}$.

(ii) There exist $c>0$ and $R>0$ such that for every $t \in[0, T], x \in H$ and every control $\gamma$ satisfying $|\gamma|>R$, then

$$
l(t, u, \gamma) \geq c\left(1+|\gamma|^{2}\right) .
$$

This square summability requirement justifies choice of the admissible controls as square integrable processes in Assumption 3.4(i): a control process which is not square summable would have infinite cost.

As stated in the introduction, to solve the control problem in the state space setting we associate to this equation a backward stochastic equation and we try to solve the control problem via this forward-backward system. Nonlinear BSDEs were first introduced by Pardoux and Peng [31]. A major application of BSDEs is in stochastic control: see, e.g., [16, 34]. Also see [23, 17] and [22].

Copyright $@$ by SIAM. Unauthorized reproduction of this article is prohibited. 
Remark 5.2. In view of other possible applications of our construction, a different kind of control problem should be addressed. In particular, a natural question is to consider an infinite time horizon. In this case, it seems necessary to strengthen the assumptions on the coefficients, so that the solution to (3.9) exists on $\mathbb{R}_{+}$. Further, a suitable modification of the forward-backward differential equation approach is required. We aim to return on the topic in a subsequent paper.

We define in a classical way the Hamiltonian function relative to the above problem: for all $t \in[0, T], v \in X_{\eta}, z \in U^{*}$,

$$
\psi(t, v, z)=\inf \{l(t, J(v), \gamma)+z r(t, J(v), \gamma): \gamma \in \Xi\}
$$

and the set of minimizers in (5.4):

$$
\Gamma(t, v, z)=\{\gamma \in \Xi: l(t, J(v), \gamma)+z r(t, J(v), \gamma)=\psi(t, v, z)\} .
$$

The map $\psi$ is a Borel measurable function from $[0, T] \times X_{\eta} \times U^{*}$ to $\mathbb{R}$. In fact, by the continuity of $r$ and $l$ with respect to $\gamma$, we have

$$
\psi(t, v, z)=\inf _{\gamma \in K}[l(t, J(v), \gamma)+z r(t, J(v), \gamma)] \quad \text { for } t \in[0, T], v \in X_{\eta}, z \in U^{*},
$$

where $K$ is any countable dense subset of $\Xi$.

Moreover, by a direct computation using the assumptions on $l$ and $r$ (see also [20, Lemma 3.1]), we can show that there exists a constant $C>0$ such that

$$
-C\left(1+|z|^{2}\right) \leq \psi(t, v, z) \leq l(t, J(v), \gamma)+C|z|(1+|\gamma|) \quad \forall \gamma \in \Xi .
$$

The existence of a minimizer for the Hamiltonian is not a direct consequence of our setting. Then, we require it explicitly.

Assumption 5.3. $\Gamma(t, v, z)$ is nonempty for all $t \in[0, T], v \in X_{\eta}$, and $z \in U^{*}$.

In several cases, this assumption can be shown to hold.

Remark 5.4. Let us consider the following situation: $H=U=\Xi=L^{2}(0,1)$, and $r(t, v, \gamma)=\gamma, l(t, u, \gamma)=q(t, u)+|\gamma|^{2} / 2$, where $q:[0, T] \times H \rightarrow \mathbb{R}$ such that $0 \leq q(t, u) \leq C\left(1+|u|^{2}\right)$. Then Assumption 5.3 holds: $\Gamma(t, v, z)=-z$ and we note that it turns out to be a continuous function of $z$ only. Moreover, we have

$$
\psi(t, v, z)=q(t, J(v))-\frac{|z|^{2}}{2} .
$$

By simple calculation (see [20, Lemma 3.1]), we can prove that if Assumption 5.3 holds, then

$\psi(t, v, z)=\min _{\gamma \in \Xi,|\gamma| \leq C(1+|v|+|z|)}[l(t, J(v), \gamma)+z r(t, J(v), \gamma)] \quad t \in[0, T], v \in X_{\eta}, z \in U^{*}$,

that is, the infimum in (5.4) is attained in a ball of radius $C(1+|v|+|z|)$, and

$$
\psi(t, v, z)<l(t, J(v), \gamma)+z r(t, J(v), \gamma) \quad \text { if }|\gamma|>C(1+|v|+|z|) .
$$

Moreover, from (5.6) it follows that for every $t \in[0, T]$ and $v \in X_{\eta}$, the map $z \rightarrow$ $\psi(t, v, z)$ is continuous on $U^{*}$.

Next we take an arbitrary, complete probability space $\left(\Omega, \mathcal{F}, \mathbb{P}^{\circ}\right)$ and a Wiener process $W^{\circ}$ in $U$ with respect to $\mathbb{P}^{\circ}$. We denote by $\left(\mathcal{F}_{t}^{\circ}\right)$ the associated Brownian 
filtration, i.e., the filtration generated by $W^{\circ}$ and augmented by the $\mathbb{P}^{\circ}$-null sets of $\mathcal{F} ;\left(\mathcal{F}_{t}^{\circ}\right)$ satisfies the usual conditions. We introduce the forward equation

$$
\begin{aligned}
\mathrm{d} v(t) & =B v(t) \mathrm{d} t+(I-B) P g(t, J(v(t))) \mathrm{d} W_{t}^{\circ}, \\
v(0) & =v_{0},
\end{aligned}
$$

whose solution is a continuous $\left(\mathcal{F}_{t}^{\circ}\right)$-adapted process, which exists and is unique by the results in section 3 . Next we consider the associated backward equation of parameters $(\psi, T, \phi(J(v(T)))$

$$
\begin{aligned}
d Y_{t} & =-\psi\left(t, v(t), Z_{t}\right) \mathrm{d} t+Z_{t} \mathrm{~d} W_{t}^{\circ}, \quad t \in[0, T], \\
Y_{T} & =\phi(J(v(t))) .
\end{aligned}
$$

The solution of (5.9) exists in the sense specified by the following result.

Proposition 5.5. Under Assumptions 2.2, 3.1, and 3.4, and 5.1, there exist Borel measurable functions $\theta$ and $\zeta$ with values in $\mathbb{R}$ and $U^{*}$, respectively,

$$
\theta:[0, T] \times X_{\eta} \rightarrow \mathbb{R} \text { and } \zeta:[0, T] \times X_{\eta} \rightarrow U^{*},
$$

with the following property: for an arbitrarily chosen complete probability space $(\Omega, \mathcal{F}$, $\left.\mathbb{P}^{\circ}\right)$ and Wiener process $W^{\circ}$ in $U$, denoting by $v$ the solution of (5.8), the processes $Y, Z$ defined by

$$
Y_{t}=\theta(t, v(t)), \quad Z_{t}=\zeta(t, v(t))
$$

satisfy

$$
\mathbb{E}^{\circ} \sup _{t \in[0, T]}\left|Y_{t}\right|^{2}<\infty, \quad \mathbb{E}^{\circ} \int_{0}^{T}\left|Z_{t}\right|^{2} \mathrm{~d} t<\infty
$$

moreover, $Y$ is continuous and nonnegative, and $\mathbb{P}^{\circ}$-a.s.,

$$
Y_{t}+\int_{t}^{T} Z_{s} \mathrm{~d} W_{s}^{\circ}=\phi(v(t))+\int_{t}^{T} \psi\left(s, v(s), Z_{s}\right) \mathrm{d} s, \quad t \in[0, T] .
$$

Finally, this solution is the maximal solution among all the solutions ( $\left.Y^{\prime}, Z^{\prime}\right)$ of (5.9) satisfying

$$
\mathbb{E}^{\circ} \sup _{t \in[0, T]}\left|Y_{t}^{\prime}\right|^{2}<\infty
$$

This theorem gives a representation of the processes $(Y ; Z)$ solution of the backward equation (5.9) as functionals of the process $v$ solution to the forward equation (5.8). The main difficulty here is to prove the existence of the solution to the backward equation. In fact the generator $\psi$ has quadratic growth with respect to $z$ and the condition at final time is unbounded. We deal with the same problem studied in [20, Proposition 2.3] and in order to solve the BSDE we use the same procedure, adopting the approximation strategy introduced in [6]. We recall that in our case the structure of the forward equation arises from the Volterra equation and has an unbounded operator in the diffusion term. Moreover, we work in an infinite dimensional setting. This fact requires particular attention to solve the BSDE. To do this we have to extend to the infinite dimensional case a monotone stability result (see Lemma 5.6) and prove explicitly its applicability to our situation. 
Before giving the proof of Proposition 5.5, we state the following lemma, which gives the main argument of the existence of the solution of (5.9). This lemma is an extension to the infinite dimensional framework, with exactly the same proofs, of the monotone stability result contained in the Lemma 2 in [7] (which is a slight generalization of Proposition 2.4 in [25]).

Lemma 5.6. Let $\tau$ be a stopping time, let $\left(F_{0}, \tau, \xi\right)$ be a set of parameters, and let $\left(F_{n}, \tau, \xi_{n}\right)_{n \geq 1}$ be a sequence of parameters of the backward equation (5.9) such that for each $n \geq 0$ the following hold:

(i) There exist, independent on $n$, two constants $\beta \geq 0 \gamma>0$ together with a progressively measurable nonnegative stochastic process $\{\alpha(t)\}_{0 \leq t \leq T}$ and a deterministic continuous nondecreasing function $\varphi: \mathbb{R}_{+} \rightarrow \mathbb{R}_{+}$with $\varphi(0)=0$ such that, $\mathbb{P}-$ a.s.,

(a) for all $t \in[0, T],(y, z) \rightarrow F_{n}(t, y, z)$ is continuous;

(b) monotonicity in $y$ : for each $(t, z) \in[0, T] \times U^{*}$,

$$
\forall y \in \mathbb{R}, \quad<y, F_{n}(t, y, z)-F_{n}(t, 0, z) \leq \beta|y|^{2} ;
$$

(c) growth condition:

$$
\forall(t, y, z) \in[0, T] \times \mathbb{R} \times U^{*},\left|F_{n}(t, y, z)\right| \leq \alpha_{t}+\varphi(|y|)+\frac{\gamma}{2}|z|^{2} .
$$

(ii) For each $n \geq 1$, the BSDE (5.9) with parameters $\left(F_{n}, \tau, \xi_{n}\right)$ has a solution $\left(Y^{n}, Z^{n}\right)$ such that $Y^{n}$ is almost surely bounded for almost every $t$, and $\mathbb{E} \int_{0}^{T}\left|Z_{t}^{n}\right|^{2} \mathrm{~d} t<$ $\infty$; the sequence $\left(Y^{n}\right)_{n}$ is monotonic, and there exists $M>0$ such that for all $n \in \mathbb{N}$, $\left\|Y^{n}\right\|_{\infty} \leq M$.

(iii) $\left(F_{n}\left(t, y_{n}, z_{n}\right)\right)_{n \geq 1}$ converges to $F_{0}(t, y, z)$ as soon as $\left(y_{n}, z_{n}\right) \rightarrow(y, z)$.

(iv) Let $\xi_{n} \in L^{\infty}(\Omega)$ be a sequence of terminal conditions converging almost surely to $\xi$.

(v) The stopping time $\tau$ is such that $\tau<\infty \mathbb{P}$-a.s.

Then there exists a pair of processes $(Y, Z)$ such that $Y$ is almost surely bounded, for almost every $t$, while

$$
\mathbb{E} \int_{0}^{T}\left|Z_{t}\right|^{2} \mathrm{~d} t<\infty
$$

and for all $T \in \mathbb{R}^{+}$

$$
\begin{aligned}
& \lim _{n \rightarrow \infty} Y^{n}=Y \text { uniformly on }[0, T] \text { in probability } \\
& \left(Z^{n}\right)_{n} \text { converges to } Z \text { in } L^{2}\left(\Omega \times[0, \tau] ; U^{*}\right)
\end{aligned}
$$

and $(Y, Z)$ is a solution of the BSDE with parameters $\left(F_{0}, \tau, \xi\right)$. In particular, if for each $n, Y^{n}$ has continuous paths, also the process $Y$ has continuous paths.

Proof of Proposition 5.5. We proceed as in [6] (compare also [20, Proposition 3.2]) in order to construct a maximal solution to

$$
\begin{aligned}
d Y_{t} & =\psi\left(t, v(t), Z_{t}\right) \mathrm{d} t+Z_{t} \mathrm{~d} W_{t}^{\circ}, \quad t \in[0, T], \\
Y_{T} & =\phi(J(v(t))) .
\end{aligned}
$$

$v$ is the solution of the forward equation (5.8), that is, a continuous $\left(\mathcal{F}_{t}^{\circ}\right)$-adapted process which exists and is unique by the results in section 3 . First we note that

$$
\mathbb{E}^{\circ} \sup _{t \in[0, T]}\|v(t)\|_{\eta}^{p}<\infty \quad \forall p \geq 2 .
$$

Copyright $@$ by SIAM. Unauthorized reproduction of this article is prohibited. 
Moreover, from (5.5) there exists a constant $C>0$ such that

$$
-C\left(1+|z|^{2}\right) \leq \psi(t, v, z) \leq l\left(t, J(v), \gamma_{0}\right)+C\left(1+\left|\gamma_{0}\right|\right)|z| .
$$

For each $n \geq C$, we define the globally Lipschitz continuous function,

$$
\psi_{n}(t, v, z)=\sup \left\{\psi(t, v, q)-n|q-z|: q \in U^{*} \cap \mathcal{H}\right\},
$$

where $\mathcal{H}$ is a numerable subset dense in $U^{*} . \psi_{n}$ is decreasing and converges to $\psi$; then by $\left(Y^{n}, Z^{n}\right)$ we denote the unique solution to the BSDE with Lipschitz coefficient $\psi_{n}$,

$$
\begin{aligned}
d Y_{t}^{n} & =-\psi_{n}\left(t, v(t), Z_{t}^{n}\right) \mathrm{d} t+Z_{t}^{n} \mathrm{~d} W_{t}^{\circ}, \quad t \in[0, T], \\
Y_{T}^{n} & =\phi(J(v(t))),
\end{aligned}
$$

and by $\left(Y^{S}, Z^{S}\right)$ the unique solution to the BSDE,

$$
\begin{aligned}
d Y_{t}^{S} & =-\left[l\left(t, J(v(t)), \gamma_{0}\right)+C\left(1+\left|\gamma_{0}\right|\right)\left|Z_{t}^{S}\right|\right] \mathrm{d} t+Z_{t}^{S} \mathrm{~d} W_{t}^{\circ}, \quad t \in[0, T], \\
Y_{T}^{S} & =\phi(J(v(t))),
\end{aligned}
$$

where $C$ is the same as in (5.5). We notice that since $\psi_{n}(t, v, 0) \geq \psi(t, v, 0) \geq 0$ and by an application of the comparison theorem (see [16]), it holds that $0 \leq Y_{t}^{n} \leq Y_{t}^{S}$. Let us introduce the following stopping times: for $k \geq 1$,

$$
\tau_{k}=\inf \left\{t \in[0, T]: \max \left(|v(t)|, Y_{t}^{S}\right)>k\right\}
$$

with the convention that $\tau_{k}=T$ if the indicated set is empty.

Then $\left(Y_{k}^{n}, Z_{k}^{n}\right):=\left(Y_{t \wedge \tau_{k}}^{n}, Z_{t \wedge \tau_{k}}^{n}\right)$ satisfies the following BSDE:

$$
Y_{k}^{n}(t)=\xi_{k}^{n}+\int_{t}^{T} 1_{s \leq \tau_{k}} \psi_{n}\left(s, v(s), Z_{k}^{n}(s)\right) \mathrm{d} s-\int_{t}^{T} Z_{k}^{n}(s) \mathrm{d} W_{s}^{\circ},
$$

where of course $\xi_{k}^{n}=Y_{k}^{n}(T)=Y_{\tau_{k}}^{n}$.

Now we fix $k$. We have in mind to use the stability property quoted in Lemma 5.6, which would imply that there exists a couple of processes $\left(Y_{k}, Z_{k}\right)$ such that $Y_{k}$ is a continuous process, $E \int_{0}^{T}\left|Z_{k}(s)\right|^{2} \mathrm{~d} s<\infty$,

$$
\begin{aligned}
& \lim _{n \rightarrow \infty} Y_{k}^{n}=Y_{k} \text { uniformly on }[0, T] \text { in probability, } \\
& \lim _{n \rightarrow \infty} \mathbb{E}^{\circ} \int_{0}^{T}\left|Z_{k}^{n}(t)-Z_{k}(t)\right|^{2} \mathrm{~d} t=0
\end{aligned}
$$

and $\left(Y_{k}, Z_{k}\right)$ solves the BSDE

$$
Y_{k}(t)=\xi_{k}+\int_{t}^{T} 1_{s \leq \tau_{k}} \psi\left(s, v(s), Z_{k}(s)\right) \mathrm{d} s-\int_{t}^{T} Z_{k}(s) \mathrm{d} W_{s}^{\circ},
$$

where $\xi_{k}=\inf _{n} Y_{\tau_{k}}^{n}$.

Claim. In our setting, the assumptions of Lemma 5.6 are satisfied.

We note that for fixed $k, Y_{k}^{n}$ is decreasing in $n$ and remains bounded by $k$. In order to apply Lemma 5.6, we have to check that for fixed $t \in\left[0, \tau_{k}\right]$ and $\omega \in \Omega$,

$$
\lim _{n \rightarrow \infty} \psi_{n}\left(t, v(t), z_{k}^{n}\right)=\psi\left(t, v(t), z_{k}\right)
$$

for each sequence $z_{k}^{n}$ converging to $z_{k}$.

Copyright (c) by SIAM. Unauthorized reproduction of this article is prohibited. 
For all $n$ we set $\bar{z}_{k}^{n}=\operatorname{argsup}\left\{\psi(t, v(t), q)-n\left|q-z_{k}^{n}\right|\right\}$ so that

$$
\psi_{n}\left(t, v(t), z_{k}^{n}\right)=\psi\left(t, v(t), \bar{z}_{k}^{n}\right)-n\left|\bar{z}_{k}^{n}-z_{k}^{n}\right|
$$

and

$$
n\left|\bar{z}_{k}^{n}-z_{k}^{n}\right|=\psi\left(t, v(t), \bar{z}_{k}^{n}\right)-\psi_{n}\left(t, v(t), z_{k}^{n}\right) \geq 0 .
$$

It follows by (5.11) and by the fact that $\psi_{n}(t, v, z) \geq \psi(t, v, z)$ that

$$
\begin{aligned}
n\left|\bar{z}_{k}^{n}-z_{k}^{n}\right|= & \psi\left(t, v(t), \bar{z}_{k}^{n}\right)-\psi_{n}\left(t, v(t), z_{k}^{n}\right) \\
\leq & l\left(t, J(v(t)), \gamma_{0}\right)+C\left(1+\left|\gamma_{0}\right|\right)\left|\bar{z}_{k}^{n}\right|+C\left(1+\left|z_{k}^{n}\right|^{2}\right) \\
\leq & C\left(1+\left.|| v(t)\right|_{\eta} ^{2}+\left|\gamma_{0}\right|^{2}\right)+C\left(1+\left|\gamma_{0}\right|\right)\left|\bar{z}_{k}^{n}-z_{k}^{n}\right| \\
& +C\left(1+\left|\gamma_{0}\right|\right)\left|z_{k}^{n}\right|+C\left(1+\left|z_{k}^{n}\right|^{2}\right) ;
\end{aligned}
$$

hence for $n>C\left(1+\left|\gamma_{0}\right|\right)$,

$$
\begin{aligned}
\left(n-C\left(1+\left|\gamma_{0}\right|\right)\right)\left|\bar{z}_{k}^{n}-z_{k}^{n}\right| \leq & C\left(1+|| v(t) \|_{\eta}^{2}+\left|\gamma_{0}\right|^{2}\right)+C\left(1+\left|\gamma_{0}\right|\right)\left|z_{k}^{n}\right| \\
& +C\left(1+\left|z_{k}^{n}\right|^{2}\right) .
\end{aligned}
$$

We state that

$$
\forall \epsilon>0 \exists n_{0} \text { s.t. } \forall n \geq n_{0} \quad\left|\bar{z}_{k}^{n}-z_{k}^{n}\right|<\epsilon,
$$

If it were not true there shall exist $\epsilon>0$ such that for all $n_{0}$ we can find $n \geq n_{0}$ with

$$
\left|\bar{z}_{k}^{n}-z_{k}^{n}\right| \geq \epsilon
$$

hence we can construct a sequence $\left\{n_{j}\right\}_{j \in \mathbb{N}}$ increasing to infinity and such that

$$
\left|\bar{z}_{k}^{n_{j}}-z_{k}^{n_{j}}\right| \geq \epsilon
$$

but then by (5.14) we get

$$
\begin{aligned}
0 & <\epsilon \leq\left|\bar{z}_{k}^{n_{j}}-z_{k}^{n_{j}}\right| \\
& \leq \frac{1}{\left(n_{j}-C\left(1+\left|\gamma_{0}\right|\right)\right)}\left[C\left(1+|v(t)|^{2}+\left|\gamma_{0}\right|^{2}\right)+C\left(1+\left|\gamma_{0}\right|\right)\left|z_{k}^{n_{j}}\right|+C\left(1+\left|z_{k}^{n_{j}}\right|^{2}\right)\right] \\
& \leq \frac{1}{\left(n_{j}-C\left(1+\left|\gamma_{0}\right|\right)\right)}\left[C\left(1+|v(t)|^{2}+\left|\gamma_{0}\right|^{2}\right)+2 C\left(1+\left|\gamma_{0}\right|\right)\left|z_{k}\right|+C\left(1+\left|z_{k}\right|^{2}\right)\right] .
\end{aligned}
$$

Now, if in the previous inequality we send $j$ to infinity we have a contradiction.

So we can conclude that $\bar{z}_{k}^{n}$ converges to $z_{k}$. It follows by (5.13) and from the definition of $\psi_{n}$

$$
\psi\left(t, v(t), \bar{z}_{k}^{n}\right) \geq \psi_{n}\left(t, v(t), z_{k}^{n}\right) \geq \psi\left(t, v(t), z_{k}^{n}\right)
$$

and by continuity of $\psi$ with respect to $z$ we get

$$
\psi\left(t, v(t), z_{k}\right) \geq \lim _{n \rightarrow \infty} \psi_{n}\left(t, v(t), z_{k}^{n}\right) \geq \psi\left(t, v(t), z_{k}\right)
$$

and the claim is proved.

Copyright $@$ by SIAM. Unauthorized reproduction of this article is prohibited. 
We return to proving Proposition 5.5. Now we fix our attention on (5.12). From the definition of $\left(Y_{k}^{n}, Z_{k}^{n}\right)$, noting that $\tau_{k} \leq \tau_{k+1}$, we have

$$
Y_{k+1}^{n}\left(t \wedge \tau_{k}\right)=Y_{k}^{n}(t), \quad Z_{k+1}^{n}(t) 1_{t \leq \tau_{k}}=Z_{k}^{n}(t) .
$$

Sending $n$ to infinity, we get

$$
Y_{k+1}\left(t \wedge \tau_{k}\right)=Y_{k}(t), \quad Z_{k+1}(t) 1_{t \leq \tau_{k}}=Z_{k}(t) .
$$

Now we define $Y$ and $Z$ on $[0, T]$ by setting

$$
Y_{t}=Y_{k}(t), \quad Z_{t}=Z_{k}(t) \text { if } t \in\left[0, \tau_{k}\right] .
$$

For $\mathbb{P}^{\circ}$-a.s. $\omega$, there exists an integer $K(\omega)$ such that for $k \geq K(\omega), \tau_{k}(\omega)=T$.

Thus $Y$ is a continuous process, $Y_{T}=\phi(v(t))$, and $\int_{0}^{T}\left|Z_{t}\right|^{2} \mathrm{~d} s<\infty \mathbb{P}^{\circ}$-a.s. From (5.12), $(Y, Z)$ satisfies

$$
Y_{t \wedge \tau_{k}}=Y_{\tau_{k}}+\int_{t \wedge \tau_{k}}^{\tau_{k}} \psi(s, v(s), Z(s)) \mathrm{d} s-\int_{t \wedge \tau_{k}}^{\tau_{k}} Z(s) \mathrm{d} W_{s}^{\circ} .
$$

By sending $k$ to infinity, we deduce that $(Y, Z)$ is a solution of $(5.10)$ and

$$
\lim _{n} \sup _{t \in[0, T]}\left|Y_{t}^{n}-Y_{t}\right|=0, \quad \lim _{n} \int_{0}^{T}\left|Z_{t}^{n}-Z_{t}\right|^{2} \mathrm{~d} t=0, \quad \mathbb{P}^{\circ} \text {-a.s. }
$$

Thus $\left|Z^{n}-Z\right|$ converges to zero in measure $\mathrm{d} \mathbb{P} \otimes \mathrm{d} t$, and passing, if needed, to a subsequence (that by abuse of language we still denote $Z^{n}$ ), we can assume that $\left|Z^{n}-Z\right| \rightarrow 0, \mathrm{~d} \mathbb{P} \otimes \mathrm{d} t$ almost everywhere.

Now, as $\psi_{n}$ is globally Lipschitz continuous, from [19] there exist Borel measurable functions

$$
\theta^{n}:[0, T] \times X_{\eta} \rightarrow \mathbb{R}, \quad \zeta^{n}:[0, T] \times X_{\eta} \rightarrow U
$$

such that

$$
Y_{t}^{n}=\theta^{n}(t, v(t)), \quad Z_{t}^{n}=\zeta(t, v(t))
$$

It suffices to define

$$
\theta(t, v)=\liminf _{n \rightarrow \infty} \theta^{n}(t, v) \quad \text { and } \quad \zeta(t, x)=\liminf _{n \rightarrow \infty} \zeta^{n}(t, x)
$$

to get

$$
Y_{t}=\theta(t, v(t)), \quad Z_{t}=\zeta(t, v(t)),
$$

which implies that $(\theta, \zeta)$ is the Borel function we look for. Finally, $0 \leq Y_{t} \leq Y_{t}^{S}$ implies that

$$
\mathbb{E} \sup _{t \in[0, T]}\left|Y_{t}\right|^{2}<\infty
$$

and from the equation

$$
\left|Y_{t}\right|^{2}+\int_{t}^{\tau_{k}}\left|Z_{s}\right|^{2} \mathrm{~d} s=2 \int_{t}^{\tau_{k}} Y_{s} \psi\left(s, v(s), Z_{s}\right) \mathrm{d} s-2 \int_{t}^{\tau_{k}} Y_{s} Z_{s} \mathrm{~d} W_{s}^{\circ},
$$

Copyright $@$ by SIAM. Unauthorized reproduction of this article is prohibited. 
taking into consideration that

$$
\begin{aligned}
Y_{s} \psi\left(s, v(s), Z_{s}\right) & \leq Y_{s}\left(l\left(s, J(v(s)), \gamma_{0}\right)+C\left(1+\left|\gamma_{0}\right|\right)\left|Z_{s}\right|\right) \\
& \leq Y_{s}^{S}\left(l\left(s, J(v(s)), \gamma_{0}\right)+C\left(1+\left|\gamma_{0}\right|\right)\left|Z_{s}\right|\right),
\end{aligned}
$$

we deduce, by standard arguments, that

$$
\mathbb{E}^{\circ} \int_{0}^{T}\left|Z_{t}\right|^{2} \mathrm{~d} t<\infty
$$

Moreover, this solution is the maximal solution among all the solutions $\left(Y^{\prime}, Z^{\prime}\right)$ satisfying

$$
\mathbb{E}^{\circ}\left[\sup _{t \in[0, T]}\left|Y_{t}^{\prime}\right|^{2}\right]<+\infty
$$

and it suffices to apply [6, Proposition 5] to deduce that $Y^{n} \geq Y^{\prime}$ and then $Y \geq$ $Y^{\prime}$.

5.1. The fundamental relation. In this section we still assume that Assumptions $2.2,3.1$, and 3.4 , and 5.1 all hold.

Proposition 5.7. Let $\theta, \zeta$ denote the functions defined in the statement of Proposition 5.5. Then for every admissible control $\gamma$ and for the corresponding trajectory $v$ starting at $v_{0}$, we have

$$
\begin{aligned}
\mathbb{J}(\gamma)= & \theta\left(0, v_{0}\right) \\
& +\mathbb{E} \int_{0}^{T}\left[-\psi(t, v(t), \zeta(t, v(t)))+\zeta(t, v(t)) \cdot r\left(t, J(v(t)), \gamma_{t}\right)+l\left(t, J(v(t)), \gamma_{t}\right)\right] \mathrm{d} t .
\end{aligned}
$$

Proof. We introduce stopping times $\tau_{n}$ and control processes $\gamma_{n}$ as in the proof of Theorem 3.5, and we denote by $v_{n}$ the solution to (3.12). Let us define

$$
W_{t}^{n}=W_{t}+\int_{0}^{t} r\left(s, J\left(v_{n}(s)\right), \gamma_{n}(s)\right) \mathrm{d} s .
$$

From the definition of $\tau_{n}$ and from (3.10), it follows that

$$
\begin{aligned}
\int_{0}^{T}\left|r\left(s, J\left(v_{n}(s)\right), \gamma_{n}(s)\right)\right|^{2} \mathrm{~d} s & \leq C \int_{0}^{T}\left(1+\left|\gamma_{n}(s)\right|\right)^{2} \mathrm{~d} s \\
& \leq C \int_{0}^{\tau_{n}}\left(1+\left|\gamma_{n}(s)\right|\right)^{2} \mathrm{~d} s+C \leq C+C n .
\end{aligned}
$$

Therefore, by defining

$$
\rho_{n}=\exp \left(\int _ { 0 } ^ { T } r \left(s, J\left(v_{n}(s)\right), \gamma_{n}(s) \mathrm{d} W_{s}-\frac{1}{2} \int_{0}^{T} \mid r\left(s, J\left(v_{n}(s)\right),\left.\gamma_{n}(s)\right|^{2} \mathrm{~d} s\right),\right.\right.
$$

the Novikov condition implies that $\mathbb{E} \rho_{n}=1$. Setting $d \mathbb{P}^{n}=\rho_{n} \mathrm{dP}$, by Girsanov's theorem $W^{n}$ is a Wiener process under $\mathbb{P}^{n}$. Let us denote by $\left(\mathcal{F}_{t}^{n}\right)$ its natural augmented filtration. Since for all $n$

$$
\begin{aligned}
\mathrm{d} v_{n}(t) & =B v_{n}(t) \mathrm{d} t+(I-B) P g\left(t, J\left(v_{n}(t)\right)\right) \mathrm{d} W_{t}^{n}, \\
v_{n}(0) & =v_{0},
\end{aligned}
$$

Copyright $\odot$ by SIAM. Unauthorized reproduction of this article is prohibited. 
has a solution by Theorem 3.3, the process $v_{n}$ is also $\mathcal{F}_{t}^{n}$ adapted. Let us define

$$
Y_{t}^{n}=\theta\left(t, v_{n}(t)\right), \quad Z_{t}^{n}=\zeta\left(t, v_{n}(t)\right) ;
$$

then, by Proposition 5.5, we have

$$
\begin{aligned}
\mathrm{d} Y_{t}^{n} & =Z_{t}^{n} \mathrm{~d} W_{t}^{n}-\psi\left(t, v_{n}(t), Z_{t}^{n}\right) \mathrm{d} t, \quad t \in[0, T], \\
Y_{T}^{n} & =\phi\left(J\left(v_{n}(T)\right)\right),
\end{aligned}
$$

and $\mathbb{E}^{n} \int_{0}^{T}\left|Z_{t}^{n}\right|^{2} \mathrm{~d} t<\infty$, where $\mathbb{E}^{n}$ denotes expectation with respect to $\mathbb{P}^{n}$. It follows that

$$
\begin{aligned}
Y_{\tau_{n}}^{n}= & \phi\left(J\left(v_{n}(T)\right)\right)+\int_{\tau_{n}}^{T} \psi\left(t, v_{n}(t), Z_{t}^{n}\right) \mathrm{d} t-\int_{\tau_{n}}^{T} Z_{t}^{n} \mathrm{~d} W_{t} \\
& -\int_{\tau_{n}}^{T} Z_{t}^{n} r\left(t, J\left(v_{n}(t)\right), \gamma_{n}(t)\right) \mathrm{d} t .
\end{aligned}
$$

We note that for every $p \in[1, \infty)$ we have

$$
\begin{aligned}
\rho_{n}^{-p}= & \exp \left(p \int_{0}^{T} r\left(s, J\left(v_{n}(s)\right), \gamma_{n}(s)\right) \mathrm{d} W_{s}^{n}-\frac{p^{2}}{2} \int_{0}^{T}\left|r\left(s, J\left(v_{n}(s)\right), \gamma_{n}(s)\right)\right|^{2} \mathrm{~d} s\right) \\
& \cdot \exp \left(\frac{p^{2}-p}{2} \int_{0}^{T}\left|r\left(s, J\left(v_{n}(s)\right), \gamma_{n}(s)\right)\right|^{2} \mathrm{~d} s\right) .
\end{aligned}
$$

By (5.15) the second exponential is bounded by a constant depending only on $n$ and $p$, while the first one has $\mathbb{P}^{n}$-expectation equal to 1 . So we conclude that $\mathbb{E}^{n} \rho_{n}^{-p}<\infty$. It follows that

$$
\begin{aligned}
\left.\left.\mathbb{E}\left(\int_{0}^{T} \mid Z_{t}^{n}\right)\right|^{2} \mathrm{~d} t\right)^{1 / 2} & \leq \mathbb{E}^{n}\left(\rho_{n}^{-2} \int_{0}^{T}\left|Z_{t}^{n}\right|^{2} \mathrm{~d} t\right)^{1 / 2} \\
& \leq\left(\mathbb{E}^{n} \rho_{n}^{-2}\right)^{1 / 2}\left(\mathbb{E}^{n} \int_{0}^{T}\left|Z_{t}^{n}\right|^{2} \mathrm{~d} t\right)^{1 / 2}<\infty
\end{aligned}
$$

and the stochastic integral in (5.17) has zero $\mathbb{P}$-expectation. Hence we obtain

$$
\mathbb{E} Y_{\tau_{n}}^{n}=\mathbb{E} \phi\left(J\left(v_{n}(T)\right)\right)+\mathbb{E} \int_{\tau_{n}}^{T}\left[\psi\left(t, v_{n}(t), Z_{t}^{n}\right)-Z_{t}^{n} r\left(t, J\left(v_{n}(t)\right), \gamma_{n}(t)\right)\right] \mathrm{d} t .
$$

Since by definition it is $\psi(t, v, z)-z r(t, J(v), \gamma)-l(t, J(v), \gamma) \leq 0$, we have

$$
\mathbb{E} Y_{\tau_{n}}^{n} \leq \mathbb{E} \phi\left(J\left(v_{n}(T)\right)\right)+\mathbb{E} \int_{\tau_{n}}^{T} l\left(t, J\left(v_{n}(t)\right), \gamma_{n}(t)\right) \mathrm{d} t .
$$

Now we let $n \rightarrow \infty$ : by the definition of $\gamma_{n}$, from (5.2) and using the fact that $J$ is bounded from $X_{\eta}$ to $H$ (see [4, Lemma 3.11]) we get

$$
\begin{aligned}
\mathbb{E} \int_{\tau_{n}}^{T} l\left(t, J\left(v_{n}(t)\right), \gamma_{n}(t)\right) \mathrm{d} t & =\mathbb{E} \int_{0}^{T} \mathbf{1}_{\left\{s>\tau_{n}\right\}} l\left(t, J\left(v_{n}(t)\right), \gamma_{0}\right) \mathrm{d} t \\
& \leq C \mathbb{E} \int_{0}^{T} \mathbf{1}_{\left\{s>\tau_{n}\right\}}\left(1+\left|J\left(v_{n}(t)\right)\right|^{2}+\left|\gamma_{0}\right|^{2}\right) \mathrm{d} s \\
& \leq C \mathbb{E}\left[\left(T-\tau_{n}\right)\left(1+\sup \left|v_{n}(t)\right|^{2}\right)\right]
\end{aligned}
$$

Copyright $@$ by SIAM. Unauthorized reproduction of this article is prohibited. 
and the right-hand side tends to 0 by the uniform integrability of $\sup _{t \in[0, T]}\left|v_{n}(t)\right|^{2}$ (see Corollary 3.6) and by (3.11).

Next we note that, again by (3.11), for $n \geq N(\omega)$ we have $\tau_{n}(\omega)=T$ and

$$
\phi\left(J\left(v_{n}(T)\right)\right)=\phi\left(J\left(v_{n}\left(\tau_{n}\right)\right)\right)=\phi\left(J\left(v\left(\tau_{n}\right)\right)\right)=\phi(J(v(t))) .
$$

We deduce, thanks to (5.3) and again to [4, Lemma 3.11], that

$$
\left|\phi\left(J\left(v_{n}(T)\right)\right)\right| \leq C\left(1+\left|v_{n}(T)\right|^{2}\right) \leq C\left(1+\sup _{t \in[0, T]}\left|v_{n}(t)\right|^{2}\right),
$$

and by the uniform integrability of $\sup _{t \in[0, T]}\left|v_{n}(t)\right|^{2}$, the right-hand side is uniformly integrable. We deduce that $\mathbb{E} \phi\left(J\left(v_{n}(T)\right)\right) \rightarrow \mathbb{E} \phi(J(v(T)))$, and from (5.18) we conclude that $\lim \sup _{n \rightarrow \infty} \mathbb{E} Y_{\tau_{n}}^{n} \leq \mathbb{E} \phi(J(v(T)))$. On the other hand, for $n \geq N(\omega)$ we have $\tau_{n}(\omega)=T$ and

$$
Y_{\tau_{n}}^{n}=Y_{T}^{n}=\phi\left(J\left(v_{n}(T)\right)\right)=\phi(J(v(T))) .
$$

Since $Y^{n}$ is positive, by an application of Fatou's lemma it follows that $\mathbb{E} \phi(J(v(T))) \leq$ $\liminf _{n \rightarrow \infty} \mathbb{E} Y_{\tau_{n}}^{n}$. We have thus proved that

$$
\lim _{n \rightarrow \infty} \mathbb{E} Y_{\tau_{n}}^{n}=\mathbb{E} \phi(J(v(T))) .
$$

Now we return to (5.16) and write

$$
Y_{\tau_{n}}^{n}=Y_{0}^{n}+\int_{0}^{\tau_{n}}-\psi\left(t, v_{n}(t), Z_{t}^{n}\right) \mathrm{d} t+\int_{0}^{\tau_{n}} Z_{t}^{n} \mathrm{~d} W_{t}+\int_{0}^{\tau_{n}} Z_{t}^{n} r\left(t, J\left(v_{n}(t)\right), \gamma_{n}(t)\right) \mathrm{d} t .
$$

Arguing as before, we conclude that the stochastic integral has zero $\mathbb{P}$-expectation. Moreover, we have $Y_{0}^{n}=\theta\left(0, v_{0}\right)$, and, for $t \leq \tau_{n}$, we also have $\gamma_{n}(t)=\gamma(t)=\gamma_{t}$, $v_{n}(t)=v(t)$, and $Z_{t}^{n}=\zeta(t, v(t))$. Thus we obtain

$$
\mathbb{E} Y_{\tau_{n}}^{n}=\theta\left(0, v_{0}\right)+\mathbb{E} \int_{0}^{\tau_{n}}\left[-\psi(t, v(t), \zeta(t, v(t)))+\zeta(t, v(t)) r\left(t, J\left(v_{t}\right), \gamma_{t}\right)\right] \mathrm{d} t,
$$

and by adding to both sides the quantity $\mathbb{E} \int_{0}^{\tau_{n}} l\left(t, J(v(t)), \gamma_{t}\right) \mathrm{d} t$ we get

$$
\begin{array}{rl}
\mathbb{E} \int_{0}^{\tau_{n}} & l\left(t, J(v(t)), \gamma_{t}\right) \mathrm{d} t+\mathbb{E} Y_{\tau_{n}}^{n}=\theta\left(0, v_{0}\right) \\
& +\mathbb{E} \int_{0}^{\tau_{n}}\left[-\psi(t, v(t), \zeta(t, v(t)))+\zeta(t, v(t)) r\left(t, J\left(v_{t}\right), \gamma_{t}\right)+l\left(t, J(v(t)), \gamma_{t}\right)\right] \mathrm{d} t .
\end{array}
$$

Noting that $-\psi(t, v(t), \zeta(t, v(t)))+\zeta(t, v(t)) r\left(t, J\left(v_{t}\right), \gamma_{t}\right)+l\left(t, J(v(t)), \gamma_{t}\right) \geq 0$ and recalling that $l(t, u, \gamma) \geq 0$, by (5.19) and the monotone convergence theorem we obtain, for $n \rightarrow \infty$,

$$
\begin{array}{rl}
\mathbb{E} \int_{0}^{T} & l\left(t, J(v(t)), \gamma_{t}\right) \mathrm{d} t+\mathbb{E} \phi(J(v(t)))=\theta\left(0, v_{0}\right) \\
& \quad+\mathbb{E} \int_{0}^{T}\left[-\psi(t, v(t), \zeta(t, v(t)))+\zeta(t, v(t)) r\left(t, J\left(v_{t}\right), \gamma_{t}\right)+l\left(t, J(v(t)), \gamma_{t}\right)\right] \mathrm{d} t,
\end{array}
$$

which gives the required conclusion.

Copyright $@$ by SIAM. Unauthorized reproduction of this article is prohibited. 
A straightforward consequence of Proposition 5.7 is the so-called verification theorem.

Corollary 5.8. For every admissible control $\gamma$ and any initial datum $v_{0}$, we have $\mathbb{J}\left(v_{0}, \gamma\right) \geq \theta\left(0, v_{0}\right)$, and the equality holds if and only if the following feedback law holds $\mathbb{P}$-a.s. for almost every $t \in[0, T]$ :

$$
\psi(t, v(t), \zeta(t, v(t)))=\zeta(t, v(t)) r\left(t, J(v(t)), \gamma_{t}\right)+l\left(t, J(v(t)), \gamma_{t}\right),
$$

where $v$ is the trajectory starting at $v_{0}$ and corresponding to the control $\gamma$.

5.2. Existence of optimal controls: The closed loop equation. The aim of this subsection is to find a weak solution (in the probabilistic sense specified in the definition below) to the so-called closed loop equation. In this section we still assume Assumptions 2.2, 3.1, 3.4, and 5.1; further, we require Assumption 5.3 to hold. Then, by the Filippov theorem (see, e.g., [2, Theorem 8.2.10, p. 316]) there exists a measurable selection of $\Gamma$, a Borel measurable function $\mu:[0, T] \times X_{\eta} \times U \rightarrow \Xi$ such that

$$
\begin{aligned}
\psi(t, v, z) & =l(t, J(v), \mu(t, v, z))+z r(t, J(v), \mu(t, v, z)) \\
t & \in[0, T], v \in X_{\eta}, z \in U^{*} .
\end{aligned}
$$

By (5.7), we have

$$
|\mu(t, v, z)| \leq C(1+|v|+|z|) .
$$

We define

$$
\bar{\gamma}(t, v)=\mu(t, v, \zeta(t, v)), \quad t \in[0, T], v \in X_{\eta},
$$

where $\zeta$ is defined in Proposition 5.5. The closed loop equation is

$$
\begin{aligned}
\mathrm{d} v(t)= & {[B v(t)+(I-B) P g(t, J(v(t))) r(t, J(v(t)), \bar{\gamma}(t, v(t)))] \mathrm{d} t } \\
& +(I-B) \operatorname{Pg}(t, J(v(t))) \mathrm{d} W_{t}, \\
v(0)= & v_{0} .
\end{aligned}
$$

Definition 5.9. A weak solution to the close loop equation (5.22) is given by the set $\left(\Omega, \mathcal{F}, \mathbb{P},\left(\mathcal{F}_{t}\right)_{t \geq 0}, W, v\right)$, where $(\Omega, \mathcal{F}, \mathbb{P})$ is a complete probability space with a filtration $\left(\mathcal{F}_{t}\right)_{t \geq 0}$ satisfying the usual conditions, $W$ is a Wiener process in $U$ with respect to $\mathbb{P}$ and $\left(\mathcal{F}_{t}\right)$, and $v$ is a continuous $\left(\mathcal{F}_{t}\right)$-adapted process with values in $H$ satisfying, $\mathbb{P}$-a.s.,

$$
\int_{0}^{T}|\bar{\gamma}(t, v(t))|^{2} \mathrm{~d} t<\infty
$$

and such that the following equality holds:

$$
\begin{aligned}
v\left(t ; v_{0}\right)= & e^{t B} v_{0}+\int_{0}^{t} e^{(t-\sigma) B}(I-B) P g(\sigma, J(v(\sigma))) r(\sigma, J(v(\sigma)), \bar{\gamma}(\sigma, v(\sigma))) \mathrm{d} \sigma \\
& +\int_{0}^{t} e^{(t-\sigma) B}(I-B) P g(\sigma, J(v(\sigma))) \mathrm{d} W(\sigma) .
\end{aligned}
$$

Proposition 5.10. Assume that Assumptions 2.2, 3.1, 3.4, and 5.1 all hold. Then there exists a weak solution of the closed loop equation, satisfying in addition

$$
\mathbb{E} \int_{0}^{T}|\bar{\gamma}(t, v(t))|^{2} \mathrm{~d} t<\infty
$$

Copyright $@$ by SIAM. Unauthorized reproduction of this article is prohibited. 
Proof. Let us take an arbitrary complete probability space $\left(\Omega, \mathcal{F}, \mathbb{P}^{\circ}\right)$ and a Wiener process $W^{\circ}$ in $U$ with respect to $\mathbb{P}^{\circ}$. Let $\left(\mathcal{F}_{t}^{\circ}\right)$ be the associated Brownian filtration. We define the process $v$ as the solution of the equation

$$
\begin{aligned}
d v(t) & =B v(t) \mathrm{d} t+(I-B) P g\left(t, J(v(t)) \mathrm{d} W_{t}^{\circ}, \quad \in t \in[0, T],\right. \\
v(0) & =v_{0} .
\end{aligned}
$$

The solution is a continuous $\left(\mathcal{F}_{t}^{\circ}\right)$-adapted process, which exists and is unique by Theorem 3.3. Moreover, it satisfies $\mathbb{E}^{\circ}\left[\sup _{t \in[0, T]}\|v(t)\|_{X_{\eta}}^{p}\right]<\infty$ for every $p \in[2, \infty)$. By Proposition 5.5, setting

$$
Y_{t}=\theta(t, v(t)) \quad Z_{t}=\zeta(t, v(t)),
$$

the following backward equation holds:

$$
\begin{aligned}
d Y_{t} & =-\psi\left(t, v(t), Z_{t}\right) \mathrm{d} t+Z_{t} \mathrm{~d} W_{t}^{\circ}, \quad t \in[0, T], \\
Y_{T} & =\phi(J(v(t))),
\end{aligned}
$$

and we have

$$
\mathbb{E}^{\circ} \int_{0}^{T}\left|Z_{t}\right|^{2} \mathrm{~d} t<\infty
$$

By (3.10) we have $|r(t, v(t), \bar{\gamma}(t, v(t)))| \leq C(1+|\bar{\gamma}(t, v(t))|)$ and, by (5.21),

$$
\begin{aligned}
|\bar{\gamma}(t, v(t))| & =|\mu(t, v(t), \zeta(t, v(t)))| \leq C(1+|v(t)|+|\zeta(t, v(t))|) \\
& =C\left(1+|v(t)|+\left|Z_{t}\right|\right) .
\end{aligned}
$$

Now let us define the family of stopping times

$$
\tau_{n}=\inf \left\{t \in[0, T]: \int_{0}^{t}|\bar{\gamma}(s, v(s))|^{2} \mathrm{~d} s>n\right\}
$$

with the convention that $\tau_{n}=T$ if the indicated set is empty. By (5.25) and (5.26), for $\mathbb{P}^{\circ}$-a.e. $\omega \in \Omega$, there exists an integer $N(\omega)$ depending on $\omega$ such that $\tau(\omega)=T$ for $n \geq N(\omega)$. Let us fix $\gamma_{0} \in \Xi$ and for every $n$ we define

$$
\begin{aligned}
\gamma_{n}(t) & =\bar{\gamma}(t, v(t))) \mathbf{1}_{\left\{t \leq \tau_{n}\right\}}+\gamma_{0} \mathbf{1}_{\left\{t \geq \tau_{n}\right\}} \\
M_{t}^{n} & =\exp \left(\int_{0}^{t} r\left(s, J(v(s)), \gamma_{n}(s)\right) \mathrm{d} W_{s}^{\circ}-\frac{1}{2} \int_{0}^{t}\left|r\left(s, J(v(s)), \gamma_{n}(s)\right)\right|^{2} \mathrm{~d} s\right) \\
M_{t} & =\left(\int_{0}^{t} r(s, J(v(s)), \bar{\gamma}(t, v(t))) \mathrm{d} W_{s}^{\circ}-\frac{1}{2} \int_{0}^{t}|r(s, J(v(s)), \bar{\gamma}(t, v(t)))|^{2} \mathrm{~d} s\right), \\
W_{t}^{n} & =W_{t}^{\circ}-\int_{0}^{t} r\left(s, J(v(s)), \gamma_{n}(s)\right) \mathrm{d} s \\
W_{t} & =W_{t}^{\circ}-\int_{0}^{t} r(s, J(v(s)), \bar{\gamma}(s, v(s))) \mathrm{d} s .
\end{aligned}
$$

By previous estimates, $M^{n}, M, W^{n}$, and $W$ are well defined; moreover,

$$
\int_{0}^{T}\left|r\left(s, J(v(s)), \gamma_{n}(s)\right)-r(s, J(v(s)), \bar{\gamma}(s, v(s)))\right|^{2} \mathrm{~d} s \rightarrow 0, \quad \mathbb{P}^{\circ} \text {-a.s. }
$$

and consequently $M_{T}^{n} \rightarrow M_{T}$ in probability and $\sup _{t \in[0, T]}\left|W_{t}^{n}-W_{t}\right| \rightarrow 0$, $\mathbb{P}^{\circ}$-a.s.

Copyright $@$ by SIAM. Unauthorized reproduction of this article is prohibited. 
We will conclude the proof by showing that there exists a probability $\mathbb{P}$ such that $W$ is a Wiener process with respect to $P$ and $\left(\mathcal{F}_{t}^{\circ}\right)$. The definition of $\tau_{n}$ and the Novikov condition imply that $\mathbb{E}^{\circ}\left[M_{T}^{n}\right]=1$. Setting $\mathrm{d} \mathbb{P}^{n}=M_{T}^{n} \mathrm{~d} \mathbb{P}^{\circ}$, by Girsanov's theorem $W^{n}$ is a Wiener process with respect to $\mathbb{P}^{n}$ and $\left(\mathcal{F}_{t}^{\circ}\right)$. Writing the backward equation with respect to $W^{n}$, we obtain

$$
Y_{\tau_{n}}=Y_{0}+\int_{0}^{\tau_{n}}-\psi\left(t, v(t), Z_{t}\right) \mathrm{d} t+\int_{0}^{\tau_{n}} Z_{t} \mathrm{~d} W_{t}^{n}+\int_{0}^{\tau_{n}} Z_{t}^{n} r\left(t, J\left(v_{t}\right), \gamma_{t}^{n}\right) \mathrm{d} t .
$$

Arguing as in the proof of Proposition 5.7, we conclude that the stochastic integral has zero expectation with respect to $\mathbb{P}^{n}$. Taking into account that $\gamma_{n}(t)=\bar{\gamma}(t, v(t))$ for $t \leq \tau_{n}$, we obtain

$$
\begin{aligned}
& \mathbb{E}^{n} Y_{\tau_{n}}+\mathbb{E}^{n} \int_{0}^{\tau_{n}} l(t, v(t), \bar{\gamma}(t, v(t))) \mathrm{d} t \\
& \quad=Y_{0}+\mathbb{E}^{n} \int_{0}^{\tau_{n}}\left[-\psi\left(t, v(t), Z_{t}\right) \mathrm{d} t+Z_{t} \cdot r\left(t, J\left(v_{t}\right), \bar{\gamma}(t, v(t))\right)+l(t, v(t), \bar{\gamma}(t, v(t)))\right] \mathrm{d} t \\
& \quad=Y_{0}
\end{aligned}
$$

with the last equality coming from the definition of $\bar{\gamma}$. Recalling that $Y$ is nonnegative, it follows that

$$
\mathbb{E}^{n} \int_{0}^{\tau_{n}} l(t, v(t), \bar{\gamma}(t, v(t))) \mathrm{d} t \leq C
$$

for some constant $C$ independent of $n$. By (5.2) we also deduce

$$
\mathbb{E}^{n} \int_{0}^{\tau_{n}}|\bar{\gamma}(t, v(t))|^{2} \mathrm{~d} t \leq C
$$

Next we prove that the family $\left(M_{T}^{n}\right)_{n \geq 1}$ is uniformly integrable by showing that $\mathbb{E}^{\circ}\left[M_{T}^{n} 1_{\left\{M_{T}^{n}>c\right\}}\right] \rightarrow 0$ as $c \rightarrow \infty$, uniformly with respect to $n$. We have

$$
\mathbb{E}^{\circ}\left[M_{T}^{n} \mathbf{1}_{\left\{M_{T}^{n}>c\right\}}\right]=\mathbb{E}^{\circ}\left[M_{T}^{n} 1_{\left\{M_{T}^{n}>c, \tau_{n}=T\right\}}\right]+\mathbb{E}^{\circ}\left[M_{T}^{n} \mathbf{1}_{\left\{M_{T}^{n}>c, \tau_{n}<T\right\}}\right] .
$$

The first term in the right-hand side tends to 0 uniformly with respect to $n$ : it is

$$
\mathbb{E}^{\circ}\left[M_{T}^{n} \mathbf{1}_{\left\{M_{T}^{n}>c, \tau_{n}=T\right\}}\right]=\mathbb{E}^{\circ}\left[M_{T} \mathbf{1}_{\left\{M_{T}>c, \tau_{n}=T\right\}}\right] \leq \mathbb{E}^{\circ}\left[M_{T} \mathbf{1}_{\left\{M_{T}>c\right\}}\right] \rightarrow 0
$$

since it holds that $\mathbb{E}^{\circ}\left[M_{T}^{n}\right]=1$ and then Fatou's lemma implies that $\mathbb{E}^{\circ}\left[M_{T}\right] \leq 1$. The second term in the right-hand side of (5.28) can be estimated as follows:

$$
\begin{aligned}
\mathbb{E}^{\circ}\left[M_{T}^{n} 1_{\left\{M_{T}^{n}>c, \tau_{n}<T\right\}}\right] & \leq \mathbb{E}^{\circ}\left[M_{T}^{n} 1_{\left\{\tau_{n}<T\right\}}\right]=\mathbb{P}^{n}\left(\tau_{n}<T\right) \\
& \leq \mathbb{P}^{n}\left(\int_{0}^{\tau_{n}}|\bar{\gamma}(t, v(t))|^{2} \mathrm{~d} t>n\right) \leq \frac{1}{n} \mathbb{E}^{n} \int_{0}^{\tau_{n}}|\bar{\gamma}(t, v(t))|^{2} \mathrm{~d} t \leq \frac{C}{n}
\end{aligned}
$$

with the last inequality coming from (5.27). The required uniform integrability follows immediately. Recalling that $M_{T}^{n} \rightarrow M_{T}$ in probability, we conclude that $\mathbb{E}^{\circ} \mid M_{T}^{n}-$ $M_{T} \mid \rightarrow 0$, and in particular $\mathbb{E}^{\circ}\left[M_{T}\right]=1$, and $M$ is a $\mathbb{P}^{\circ}$-martingale. Thus we can define a probability $\mathbb{P}$ by setting $\mathrm{d} P=M_{T} \mathrm{~d} \mathbb{P}^{\circ}$ and by Girsanov's theorem we conclude that $W$ is a Wiener process with respect to $\mathbb{P}$ and $\left(\mathcal{F}_{t}^{\circ}\right)$. 
It remains to prove (5.24). We define the stopping times

$$
\sigma_{n}=\inf \left\{t \in[0, T]: \int_{0}^{t}\left|Z_{s}\right|^{2} \mathrm{~d} s>n\right\}
$$

with the convention that $\sigma_{n}=T$ if the indicated set is empty. Writing the backward equation with respect to $W$, we obtain

$$
Y_{\sigma_{n}}=Y_{0}-\int_{0}^{\sigma_{n}} \psi\left(t, v(t), Z_{t}\right) \mathrm{d} t+\int_{0}^{\sigma_{n}} Z_{t} \mathrm{~d} W_{t}+\int_{0}^{\sigma_{n}} Z_{t} r\left(t, J\left(v_{t}\right), \bar{\gamma}(t, v(t))\right) \mathrm{d} t,
$$

from which we deduce that

$$
\begin{aligned}
& \mathbb{E}^{n} Y_{\sigma_{n}}+\mathbb{E}^{n} \int_{0}^{\sigma_{n}} l(t, v(t), \bar{\gamma}(t, v(t))) \mathrm{d} t \\
& =Y_{0}+\mathbb{E}^{n} \int_{0}^{\sigma_{n}} \begin{array}{l}
{\left[-\psi\left(t, v(t), Z_{t}\right)+Z_{t} \cdot r(t, J(v(t)), \bar{\gamma}(t, v(t)))\right.} \\
\quad+l(t, J(v(t)), \bar{\gamma}(t, v(t)))] \mathrm{d} t
\end{array} \\
& =Y_{0}
\end{aligned}
$$

with the last equality coming from the definition of $\bar{\gamma}$. Recalling that $Y$ is nonnegative, it follows that

$$
\mathbb{E} \int_{0}^{\sigma_{n}} l(t, J(v(t)), \bar{\gamma}(t, v(t))) \mathrm{d} t \leq C
$$

for some constant $C$ independent of $n$. By (5.2) and by sending $n$ to infinity, we finally prove (5.24).

Corollary 5.11. By Corollary 5.8 it immediately follows that if $v$ is a solution to (5.22) and we set $\gamma_{s}^{*}=\bar{\gamma}\left(s, v_{s}\right)$, then $\mathbb{J}\left(v_{0}, \gamma^{*}\right)=\theta\left(0, v_{0}\right)$, and consequently $v$ is an optimal state, $\gamma_{s}^{*}$ is an optimal control, and $\bar{\gamma}$ is an optimal feedback.

Now we prove uniqueness in law for the closed loop equation. We remark that condition (5.23) is part of our definition of a weak solution.

Proposition 5.12. Under Assumptions 2.2, 3.1, 3.4, and 5.1, fix $\mu:[0, T] \times X_{\eta} \times$ $U^{*} \rightarrow \Xi$ satisfying (5.20) (and consequently (5.21)) and let $\bar{\gamma}(t, v)=\mu(t, v, \zeta(t, v)$ ). Then the weak solution of the closed loop equation (5.22) is unique in law.

Proof. Let $\left(\Omega, \mathcal{F},\left(\mathcal{F}_{t}\right)_{t \in[0, T]}, \mathbb{P},\left(W_{t}\right)_{t \in[0, T]},(v(t))_{t \in[0, T]}\right)$ be a weak solution of (5.22). Let us define

$$
\begin{aligned}
& M_{T}=\exp \left(-\int_{0}^{T} r(s, v(s), \bar{\gamma}(s, v(s))) \mathrm{d} W_{s}-\frac{1}{2} \int_{0}^{T}|r(s, v(s), \bar{\gamma}(s, v(s)))|^{2} \mathrm{~d} s\right), \\
& W_{t}^{\circ}=W_{t}+\int_{0}^{T} r(s, v(s), \bar{\gamma}(s, v(s))) \mathrm{d} s .
\end{aligned}
$$

By (3.10) and (5.23), $M_{T}$ and $W^{\circ}$ are well defined. We claim that $\mathbb{E} M_{T}=1$. Assuming the claim for a moment, and setting $\mathrm{d} P^{\circ}=M_{T} \mathrm{~d} \mathbb{P}$, we know by Girsanov's theorem that $W^{\circ}$ is a Wiener process under $P^{\circ}$, and further $v$ solves

$$
\begin{aligned}
\mathrm{d} v(t) & =B v(t) \mathrm{d} t+(I-B) P g(t, J(v(t))) \mathrm{d} W_{t}^{\circ}, \\
v(0) & =v_{0},
\end{aligned}
$$

Copyright $@$ ㅇ by SIAM. Unauthorized reproduction of this article is prohibited. 
and

$$
M_{T}=\exp \left(-\int_{0}^{T} r(s, v(s), \bar{\gamma}(s, v(s))) \mathrm{d} W_{s}^{\circ}+\frac{1}{2} \int_{0}^{T}|r(s, v(s), \bar{\gamma}(s, v(s)))|^{2} \mathrm{~d} s\right) .
$$

By Theorem 3.3 the law of $\left(v, W^{\circ}\right)$ under $P^{\circ}$ is uniquely determined by $g$ and $v_{0}$. Taking into account the last displayed formula, we conclude that the law of $\left(v, W^{\circ}, M_{T}\right)$ under $P^{\circ}$ is also uniquely determined, and consequently so is the law of $v$ under $\mathbb{P}$.

To conclude the proof it remains to show that $\mathbb{E}\left[M_{T}\right]=1$. We define the stopping times

$$
\tau_{n}=\inf \left\{t \in[0, T]: \int_{0}^{t}|\bar{\gamma}(s, v(s))|^{2} \mathrm{~d} s>n\right\}
$$

with the convention that $\tau_{n}=T$ if the indicated set is empty. By (5.23), for $\mathbb{P}$-almost every $\omega \in \Omega$ there exists an integer $N(\omega)$ depending on $\omega$ such that $\tau_{n}(\omega)=T$ for $n \geq N(\omega)$.

Let us fix $\gamma_{0} \in \Xi$ and let us define, for every $n \in \mathbb{N}$,

$$
\begin{aligned}
\gamma_{n}(t) & =\bar{\gamma}(t, v(t)) 1_{t \leq \tau_{n}}+\gamma_{0} 1_{t \geq \tau_{n}}, \\
M_{T}^{n} & =\exp \left(-\int_{0}^{T} r\left(s, J\left(v_{n}(s)\right), \gamma_{n}(s)\right) \mathrm{d} W_{s}-\frac{1}{2} \int_{0}^{T}\left|r\left(s, J\left(v_{n}(s)\right), \gamma_{n}(s)\right)\right|^{2} \mathrm{~d} s\right) .
\end{aligned}
$$

By (3.10) and the definition of $\tau_{n}$, the Novikov condition shows that $\mathbb{E} M_{T}^{n}=1$. Moreover, we have

$$
\int_{0}^{T}\left|r\left(s, v(s), \gamma_{n}(s)\right)-r(s, v(s), \bar{\gamma}(s, v(s)))\right|^{2} \mathrm{~d} s \rightarrow 0, \quad \mathbb{P} \text {-a.s. },
$$

and consequently $M_{T}^{n} \rightarrow M_{T}$ in probability. In order to conclude the proof it is therefore enough to show that the family $\left(M_{T}^{n}\right)_{n \geq 1}$ is uniformly integrable. To prepare for this, let us set $d \mathbb{P}^{n}=M_{T}^{n} \mathrm{~d} \mathbb{P}$ and note that by Girsanov's theorem, the process $W_{t}^{n}=W_{t}+\int_{0}^{t} r\left(s, v(s), \gamma_{s}^{n}\right) \mathrm{d} s$ is a Wiener process under $\mathbb{P}^{n}$. Since $v$ solves

$$
\begin{aligned}
\mathrm{d} v(t) & =B v(t) \mathrm{d} t+(I-B) P g(t, J(v(t))) \mathrm{d} W_{t}^{n}, \quad t \in[0, T], \\
v(0) & =v_{0},
\end{aligned}
$$

it follows that $v$ is adapted to the Brownian filtration $\left(\mathcal{F}_{t}^{n}\right)$ associated to $W^{n}$, and its law under $\mathbb{P}^{n}$ is uniquely determined by $g$ and $v_{0}$. In particular, the quantities

$$
C^{\prime}:=\mathbb{E}^{n} \int_{0}^{T}|v(t)|^{2} \mathrm{~d} t, \quad C^{\prime \prime}:=\mathbb{E}^{n} \int_{0}^{T}|\zeta(t, v(t))|^{2} \mathrm{~d} t
$$

do not depend on $n$ (here $\mathbb{E}^{n}$ denotes, of course, the expectation with respect to $\left.\mathbb{P}^{n}\right) . C^{\prime}$ is clearly finite. By Proposition 5.5, setting $Z_{t}=\zeta(t, v(t))$, we have $\mathbb{E}^{n} \int_{0}^{T}$ $|\zeta(t, v(t))|^{2} \mathrm{~d} t=\mathbb{E}^{n} \int_{0}^{T}\left|Z_{t}\right|^{2} \mathrm{~d} t<\infty$, and it follows that $C^{\prime \prime}$ is also finite. Now let us prove the uniform integrability of the family $\left(M_{T}^{n}\right)_{n \geq 1}$ by showing that that $\mathbb{E}\left[M_{T}^{n} 1_{\left\{M_{T}^{n}>c\right\}}\right] \rightarrow 0$ as $c \rightarrow \infty$, uniformly with respect to $n$. We have

$$
\mathbb{E}\left[M_{T}^{n} \mathbf{1}_{\left\{M_{T}^{n}>c\right\}}\right]=\mathbb{E}\left[M_{T}^{n} \mathbf{1}_{\left\{M_{T}^{n}>c, \tau_{n}=T\right\}}\right]+\mathbb{E}\left[M_{T}^{n} 1_{\left\{M_{T}^{n}>c, \tau_{n}<T\right\}}\right] .
$$

Copyright (c) by SIAM. Unauthorized reproduction of this article is prohibited. 
The first term in the right-hand side tends to 0 uniformly with respect to $n$, since

$$
\mathbb{E}\left[M_{T}^{n} \mathbf{1}_{\left\{M_{T}^{n}>c, \tau_{n}=T\right\}}\right]=\mathbb{E}\left[M_{T} \mathbf{1}_{\left\{M_{T}>c, \tau_{n}=T\right\}}\right] \leq \mathbb{E}\left[M_{T} \mathbf{1}_{\left\{M_{T}>c\right\}}\right] \rightarrow 0,
$$

due to the fact that the equality $\mathbb{E}\left[M_{T}^{n}\right]=1$ and Fatou's lemma imply that $\mathbb{E}\left[M_{T}\right] \leq 1$. The second term in the right-hand side of (5.29) can be estimated as follows:

$$
\begin{aligned}
\mathbb{E}\left[M_{T}^{n} \mathbf{1}_{\left\{M_{T}^{n}>c, \tau_{n}<T\right\}}\right] \leq & \mathbb{E}\left[M_{T}^{n} \mathbf{1}_{\left\{\tau_{n}<T\right\}}\right]=\mathbb{P}^{n}\left(\tau_{n}<T\right) \\
& \leq \mathbb{P}^{n}\left(\int_{0}^{\tau_{n}}|\bar{\gamma}(t, v(t))|^{2} \mathrm{~d} t>n\right) \leq \frac{1}{n} \mathbb{E}^{n} \int_{0}^{\tau_{n}}|\bar{\gamma}(t, v(t))|^{2} \mathrm{~d} t .
\end{aligned}
$$

By (5.21) we have

$$
|\bar{\gamma}(t, v(t))|^{2}=|\mu(t, v(t), \zeta(t, v(t)))|^{2} \leq C\left(1+|v(t)|^{2}+|\zeta(t, v(t))|^{2}\right)
$$

for some constant $C$, and it follows that

$$
\mathbb{E}\left[M_{T}^{n} \mathbf{1}_{\left\{M_{T}^{n}>c, \tau_{n}<T\right\}}\right] \leq \frac{C}{n} \mathbb{E}^{n} \int_{T}^{0}\left(1+|v(t)|^{2}+|\zeta(t, v(t))|^{2}\right) \mathrm{d} t=\frac{C}{n}\left(T+C^{\prime}+C^{\prime \prime}\right)
$$

with $C^{\prime}$ and $C^{\prime \prime}$ defined as above. The required uniform integrability follows immediately and this completes the proof.

5.3. Application to the motivating example. We wish to apply the previous results to perform the synthesis of the optimal control described in section 2.1: minimize the cost functional

$$
\mathbb{J}\left(\theta_{0}, \gamma\right)=\mathbb{E} \int_{0}^{T}\left[\frac{1}{2}|\gamma(t)|_{\mathbb{R}^{d}}^{2}+\int_{\mathcal{O}}|\theta(t, x)|^{2} \mathrm{~d} x\right] \mathrm{d} t+\mathbb{E} \int_{\mathcal{O}}|\theta(T, x)|^{2} \mathrm{~d} x
$$

subject to

$$
\frac{\partial}{\partial t} \int_{-\infty}^{t} c(t-s) \theta(s, x) \mathrm{d} s-k_{0} \Delta \theta(t, x)=\left[\sum_{i}^{d} \sigma_{j}(\theta(t, x)) \gamma_{j}(t)+\sum_{i}^{d} \sigma_{j}(\theta(t, x)) d \beta_{j}(t)\right] .
$$

The admissible controls are all processes $\gamma=\left(\gamma_{1}, \ldots, \gamma_{d}\right)$ in $L^{2}\left(\Omega \times[0, T] ; \mathbb{R}^{d}\right)$.

The above problem falls under the scope of the general results proved in this section letting $H=L^{2}(\mathcal{O}), U=\Xi=\mathbb{R}^{d}$ and $W_{t}=\left(\beta_{1}(t), \cdot, \cdot, \cdot, \beta_{d}(t)\right), t \geq 0$. We define the coefficient $g: H \rightarrow L_{2}(U, H)$ as

$$
(g(\theta) z)(x)=\sum_{i}^{d} z_{j} \sigma_{j}(\theta(x)), \quad z \in \mathbb{R}^{d} .
$$

We take $r$ to be the identity operator.

With the above definitions, our problem can be written in the following form: minimize

$$
\mathbb{J}\left(\theta_{0}, \gamma\right)=\mathbb{E} \int_{0}^{T}\left[\frac{1}{2}|\gamma(t)|^{2}+|\theta(t)|^{2}\right] \mathrm{d} t+\mathbb{E}|\theta(T)|^{2}
$$

subject to

$$
\frac{\partial}{\partial t} \int_{-\infty}^{t} c(t-s) \theta(s) \mathrm{d} s-k_{0} \Delta \theta(t)=g(\theta(t))[\gamma(t)+\dot{W}(t)] .
$$

Copyright $@$ ㅇ by SIAM. Unauthorized reproduction of this article is prohibited. 
We note that in our case the function $l$ in the running cost is given by

$$
l(\theta, \gamma)=\frac{1}{2}|\gamma|^{2}+|\theta|^{2},
$$

while the function $\phi$ in the final cost is

$$
\phi(\theta)=|\theta|^{2} ;
$$

hence they satisfy Assumption 5.1. Then Proposition 5.10 and Corollary 5.11 can be applied and we obtain a characterization of the optimal control by a feedback law.

\section{REFERENCES}

[1] O. P. Agrawal, Solution for a fractional diffusion-wave equation defined in a bounded domain, Nonlinear Dynam., 29 (2002), pp. 145-155.

[2] A. Ambrosetti and G. Prodi, A primer of nonlinear analysis, Cambridge Stud. Adv. Math. 34. Cambridge University Press, Cambridge, UK, 1995.

[3] S. Bonaccorsi, G. Da Prato, and L. Tubaro, Asymptotic behavior of a class of nonlinear stochastic heat equations with memory effects, SIAM J. Math. Anal., to appear.

[4] S. Bonaccorsi and W. Desch, Volterra Equations Perturbed by Noise, Technical report UTM 698, Matematica, University of Trento, 2006; also available online from http://eprints. biblio.unitn.it/archive/00001021/.

[5] S. BonacCorsi And E. Mastrogiacomo, An analytic approach to stochastic Volterra equations with completely monotone kernels, J. Evol. Equ., 9 (2009), pp. 315-339.

[6] P. BRIAND AND Y. Hu, BSDE with quadratic growth and unbounded terminal value, Probab. Theory Related Fields, 136 (2006), pp. 604-618.

[7] P. BRIAND AND Y. Hu, Quadratic BSDEs with convex generators and unbounded terminal conditions, Probab. Theory Related Fields, 141 (2008), pp. 543-567.

[8] P. Clément, G. Da Prato, and J. Prüss, White noise perturbation of the equations of linear parabolic viscoelasticity, Rend. Istit. Mat. Univ. Trieste, 29 (1997), pp. 207-220.

[9] B. Coleman and M. Gurtin, Equipresence and constitutive equations for rigid heat conductors, Z. Angew. Math. Phys., 18 (1967), pp. 199-208.

[10] G. DA PRATO AND J. ZABCZYK, Evolution equations with white-noise boundary conditions, Stoch. Stoch. Rep., 42 (1993), pp. 167-182.

[11] G. Da Prato and J. Zabczyk, Ergodicity for Infinite Dimensional Systems, Cambridge University Press, Cambridge, UK, 1996.

[12] G. Da Prato and J. Zabczyk, Stochastic Equations in Infinite Dimensions, Cambridge University Press, Cambridge, UK, 1992.

[13] A. Debussche, M. Fuhrman, and G. Tessitore, Optimal control of a stochastic heat equation with boundary-noise and boundary-control, ESAIM Control Optim. Calc. Var., 13 (2007), pp. $178-205$.

[14] W. Desch AND R. K. Miller, Exponential stabilization of Volterra integral equations with singular kernels, J. Integral Equations Appl., 1 (1988), pp. 397-433.

[15] K. J. Engel ANd R. NAGEL, One-Parameter Semigroups for Linear Evolution Equations, Graduate Texts in Math. 194, Springer-Verlag, Berlin, 2000.

[16] N. El Karoui, S. Peng, And M. C. Quenez, Backward stochastic differential equations in finance, Math. Finance, 7 (1997), pp. 1-71.

[17] N. El Karoui and S. Hamadène, BSDEs and risk-sensitive control, zero-sum and nonzerosum game problems of stochastic functional differential equations, Stochastic Process. Appl., 107 (2003), pp. 145-169.

[18] G. FABBRI AND B. Goldys, An LQ problem for the heat equation on the halfline with Dirichlet boundary control and noise, SIAM J. Control Optim., 48 (2009), pp. 1473-1488.

[19] M. Fuhrman, A class of stochastic optimal control problems in Hilbert spaces: BSDEs and optimal control laws, state constraints, conditioned processes, Stochastic Process. Appl., 108 (2003), pp. 263-298.

[20] M. Fuhrman, Y. Hu, and G. Tessitore, On a class of stochastic optimal control problems related to BSDEs with quadratic growth, SIAM J. Control Optim., 45 (2006), pp. 12791296.

[21] M. Fuhrman and G. Tessitore, Nonlinear Kolmogorov equations in infinite dimensional spaces: The backward stochastic differential equations approach and applications to optimal control, Ann. Probab., 30 (2002), pp. 1397-1465.

Copyright (c) by SIAM. Unauthorized reproduction of this article is prohibited. 
[22] M. Fuhrman And G. Tessitore, Existence of optimal stochastic controls and global solutions of forward-backward stochastic differential equations, SIAM J. Control Optim., 43 (2004), pp. 813-830.

[23] S. Hamadène And J.-P. LePeltier, Backward equations, stochastic control and zero-sum stochastic differential games, Stoch. Stoch. Rep., 54 (1995), pp. 221-231.

[24] K. W. Homan, An Analytic Semigroup Approach to Convolution Volterra Equations, Ph.D. thesis, Delft University Press, Delft, The Netherlands, 2003.

[25] Y. Kobylanski, Backward stochastic differential equations and partial differential equations with quadratic growth, Ann. Probab., 28 (2000), pp. 558-602.

[26] F. MASIERO, A stochastic optimal control problem for the heat equation on the halfine with Dirichlet boundary-noise and boundary-control, Appl. Math. Optim., 62 (2010), pp. 253294.

[27] B. MAslowski, Stability of semilinear equations with boundary and pointwise noise, Ann. Scuola Norm. Sup. Pisa Cl. Sci., 22 (1995), pp. 55-93.

[28] R. K. MilleR, Linear Volterra integrodifferential equations as semigroups, Funkcial. Ekvac., 17 (1974), pp. 39-55.

[29] J. Nunziato, On heat conduction in materials with memory, Quart. Appl. Math., 29 (1971), pp. 187-204.

[30] E. PARDoux, Nonlinear filtering, prediction and smoothing equations, Stochastics, 6 (1981/82), pp. 193-231.

[31] E. Pardoux And S. Peng, Adapted solution of a backward stochastic differential equation, Systems Control Lett., 14 (1990), pp. 55-61.

[32] J. Prüss, Evolutionary Integral Equations and Applications, Monogr. Math. 87, Birkhäuser, Basel, 1993.

[33] R. B. Sowers, Multidimensional reaction-diffusion equations with white noise boundary perturbations, Ann. Probab., 22 (1994), pp. 2071-2121.

[34] J. Yong And X. Y. Zhou, Stochastic controls, in Hamiltonian Systems and HJB Equations, Appl. Math. 43, Springer-Verlag, New York, 1999.

Copyright (c) by SIAM. Unauthorized reproduction of this article is prohibited. 